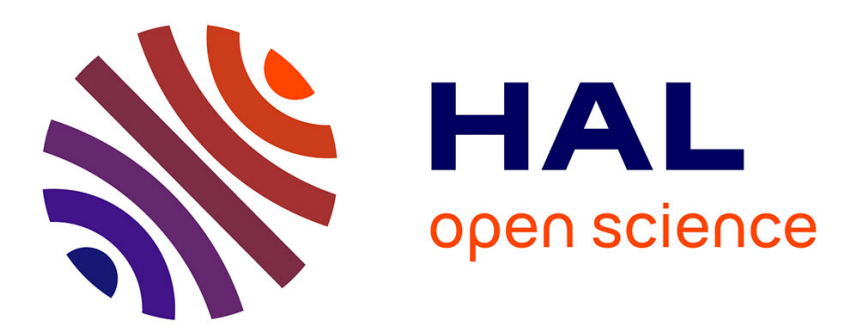

\title{
A velocity decomposition method for exergy-based drag prediction
}

\author{
Miguel Angel Aguirre, Sébastien Duplaa, Xavier Carbonneau, Andrew \\ Turnbull
}

\section{- To cite this version:}

Miguel Angel Aguirre, Sébastien Duplaa, Xavier Carbonneau, Andrew Turnbull. A velocity decomposition method for exergy-based drag prediction. AIAA Journal, 2020, 58 (11), pp.4686-4701. 10.2514/1.J059414 . hal-03099924

\section{HAL Id: hal-03099924 \\ https://hal.science/hal-03099924}

Submitted on 6 Jan 2021

HAL is a multi-disciplinary open access archive for the deposit and dissemination of scientific research documents, whether they are published or not. The documents may come from teaching and research institutions in France or abroad, or from public or private research centers.
L'archive ouverte pluridisciplinaire HAL, est destinée au dépôt et à la diffusion de documents scientifiques de niveau recherche, publiés ou non, émanant des établissements d'enseignement et de recherche français ou étrangers, des laboratoires publics ou privés. 


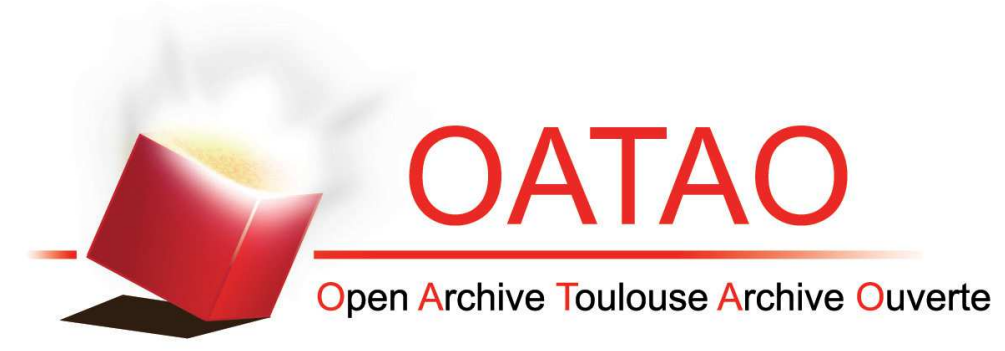

\section{Open Archive Toulouse Archive Ouverte (OATAO)}

OATAO is an open access repository that collects the work of some Toulouse researchers and makes it freely available over the web where possible.

This is an author's version published in: https://oatao.univ-toulouse.fr/27141

Official URL:https://doi.org/10.2514/1.J059414

\section{To cite this version :}

Aguirre, Miguel Angel and Duplaa, Sébastien and Carbonneau, Xavier and Turnbull, Andrew A velocity decomposition method for exergy-based drag prediction. (2020) AIAA Journal, 58 (11). 4686-4701. ISSN 0001-1452

Any correspondence concerning this service should be sent to the repository administrator: tech-oatao@listes-diff.inp-toulouse.fr 


\title{
Velocity Decomposition Method for Exergy-Based Drag Prediction
}

\author{
Miguel Angel Aguirre* \\ Safran Tech, 78117, Magny-les-Hameaux, France \\ Sébastien Duplaa $\ddagger$ and Xavier Carbonneau \\ ISAE-SUPAERO, Toulouse University, 31055 Toulouse, France \\ and \\ Andrew Turnbull $\stackrel{\S}{-}$ \\ Safran Tech, 78117, Magny-les-Hameaux, France \\ https://doi.org/10.2514/1.J059414
}

\begin{abstract}
The exergy method is a powerful tool for aerodynamic analysis and drag prediction. However, its formulation still requires further improvements in order to obtain a useful drag breakdown for the analysis of wind-tunnel data (like the far-field methods). The far-field drag breakdown is achieved by using a velocity decomposition technique, but the related formulation is not well suited for the exergy method. Thus, the main objective of this work is to develop a new velocity decomposition suited for the exergy analysis and to propose a related exergy-based drag breakdown formulation for wind-tunnel applications.
\end{abstract}

\section{Nomenclature}

$\dot{\mathcal{A}}=$ total anergy outflow rate, $\mathrm{W}$

$a=$ speed of sound, $\mathrm{m} \cdot \mathrm{s}^{-1}$

$C_{D}=$ drag coefficient

$c \quad=\quad$ airfoil chord, $\mathrm{m}$

$c_{v} \quad=$ mass specific heat at constant volume, $\mathrm{J} \cdot \mathrm{kg}^{-1} \cdot \mathrm{K}^{-1}$

$D=$ drag force, $\mathrm{N}$

$\dot{E}_{p} \quad=$ pressure exergy, $\mathrm{W}$

$\dot{E}_{u} \quad=$ axial kinetic exergy, $\mathrm{W}$

$\dot{E}_{v} \quad=$ transverse kinetic exergy, $\mathrm{W}$

$e \quad=\quad$ mass specific internal energy, $\mathrm{J} \cdot \mathrm{kg}^{-1}$

$h_{t} \quad=$ mass specific total enthalpy, $\mathrm{J} \cdot \mathrm{kg}^{-1}$

$\boldsymbol{i}, \boldsymbol{j}, \boldsymbol{k}=$ unit vectors along the aerodynamic $x, y$, and $z$ axes

$M=$ Mach number $\left(u_{0} / a_{0}\right)$

$\boldsymbol{n}=n_{x} \boldsymbol{i}, n_{y} \boldsymbol{j}, n_{z} \boldsymbol{k}$, local surface normal

$P_{s}, P_{t}=$ static and total pressures, $\mathrm{Pa}$

Re $=$ Reynolds number $\left(\rho_{0} u_{0} c / \mu_{0}\right)$

$S \quad=\quad$ surface, $\mathrm{m}^{2}$

$s \quad=\quad$ mass specific entropy, $\mathrm{J} \cdot \mathrm{kg}^{-1} \cdot \mathrm{K}^{-1}$

$T_{s}, T_{t}=$ static and total temperatures, $\mathrm{K}$

$\boldsymbol{V}=u \boldsymbol{i}, v \boldsymbol{j}, w \boldsymbol{k}$, local velocity vector, $\mathrm{m} \cdot \mathrm{s}^{-1}$

$\alpha=$ angle of attack, deg

$\beta=$ angle of sideslip, deg

$\gamma=$ ratio of specific heats

$\delta()=()-()_{0}$; local variation of a parameter respect to the upstream value

$\dot{\varepsilon}_{m}=$ mechanical exergy outflow rate across the survey plane, W

$\dot{\varepsilon}_{\mathrm{th}}=$ thermal exergy outflow rate, $\mathrm{W}$

$\theta=$ elevation angle, $\mathrm{deg}$

$\begin{array}{ll}\mu & = \\ \xi & =\end{array}$ dynamic viscosity, $\mathrm{kg} \cdot \mathrm{m}^{-1} \cdot \mathrm{s}^{-1}$

axial vorticity, $\mathrm{s}^{-1}$

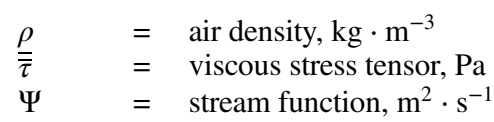

\section{Subscripts}

$b \quad=$ body

ref $\quad=$ reference

out $=$ outlet section

$p \quad=$ profile drag

$w \quad=$ wake

$0=$ upstream values

Superscripts

$* \quad=\quad$ isentropic (inviscid) component

$\overline{0}=$ nonisentropic (viscous) component

\section{Introduction}

$\mathbf{T}$ HE characteristic curves of airfoils, wings, or any other body are classically obtained by computational fluid dynamics (CFD) or wind-tunnel testing. In a CFD environment, near-field and far-field methods [1,2] have become a standard analysis tools for both the industry and the research domains. Wind-tunnel testing is more demanding for the analysis since a limited amount of parameters and measurements points can be obtained during a wind-tunnel run. This has led to the development of far-field formulations where only wake data are required to perform drag calculations [3-7].

Another powerful and promising method is the exergy analysis. This technique has been used during the last 20 years for external aerodynamic analysis, mostly in CFD and analytical applications [8-21]. Particularly, the formulation proposed by Arntz [13] represents a major milestone in the development of the exergy method. Since it requires integrating data in an infinite survey plane, it is well suited for CFD analysis, but this prevents its direct application in a wind-tunnel environment. To reduce the exergy calculations to the wake region only, a major reformulation must be made. Very few works attempting to calculate the exergy parameters by only measuring the wake parameters have been published [15-21]. Nevertheless, it still lacks a formulation directly related to the Arntz method and capable of providing all the exergy and anergy components inside the wake. Hence, the present work proposes a solution to this problem by adapting a technique already used by the far-field method to reduce data to the wake: the velocity decomposition technique. 


\section{Review of the Aerodynamic Assessment Methods}

First, the system of reference will be described; then, the straightforward near-field method is presented, followed by the wind-tunnel most-suited formulation, and finally the exergy method.

\section{A. System of Reference}

The reference system used hereafter is shown in Fig. 1. It has the $x$ axis aligned with the upstream flow direction and pointing rearward; the $y$ axis points toward the right-hand side of the body, and the $z$ axis points upward. Moreover, when control volume formulations are used, it is assumed that the outlet section $S_{\text {out }}$ of the control volume is a plane (called the "survey plane"), and it is placed normal to the $x$ axis. Also, the lateral surfaces are considered parallel to the upstream direction and far away from the body.

\section{B. Near-Field Method}

The near-field method is the classical approach used in order to obtain the total drag force $\mathrm{D}$ that is acting upon a body $[1,2]$. It takes into account the pressure and viscous forces:

$$
D=\int_{S_{b}}\left(P_{s} \boldsymbol{n}_{x}-\overline{\bar{\tau}} \cdot \boldsymbol{n}_{x}\right) \mathrm{d} S_{b}
$$

\section{Far-Field Method}

The far-field method applies the momentum conservation equation to a control volume surrounding the body. Several variants of this method are available [3-7], enabling the extraction of the drag force by only analyzing the wake of a body. This paper only uses Meheut's method [7] because it is the most accurate for the wind-tunnel measurement of stationary flows. It is based on the small perturbations method and the decomposition of the axial velocity deficit inside the wake. This leads to the following profile drag equation:

$$
\begin{aligned}
D_{p \text { Meheut }}= & \frac{\rho_{0} u_{0}^{2}}{2} \int_{S_{w}}\left[-\frac{2}{\gamma M_{0}^{2}} \Delta P_{t}-\Delta T_{t}+\left(1-\frac{M_{0}^{2}}{4}\right) \Delta T_{t}^{2}\right. \\
& \left.-\Delta P_{t} \Delta T_{t}-\left(1-M_{0}^{2}\right)\left(\Delta \bar{u}^{2}+2 \Delta u^{*} \Delta \bar{u}\right)\right] \mathrm{d} S
\end{aligned}
$$

where

$$
\begin{gathered}
\Delta P_{t}=\frac{P_{t}}{P_{t_{0}}}-1 \\
\Delta T_{t}=\frac{T_{t}}{T_{t_{0}}}-1 \\
\Delta u=\frac{u}{u_{0}}-1
\end{gathered}
$$

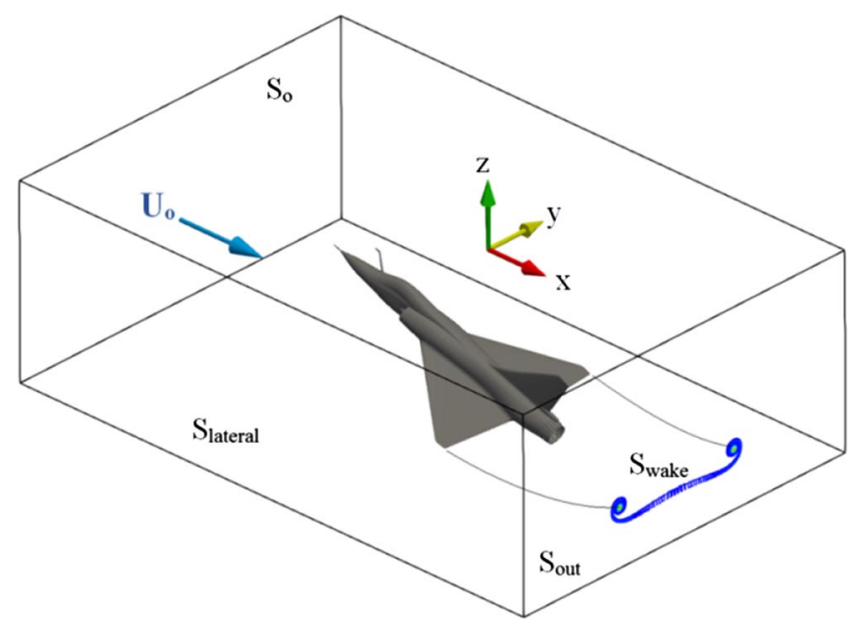

Fig. 1 Conventional reference frame.

$$
\begin{gathered}
\Delta u^{*}=\sqrt{1-\frac{2}{(\gamma-1) M_{0}^{2}}\left(\left(\frac{P_{s}}{P_{s_{0}}}\right)^{(\gamma-1) / \gamma}-1\right)-\frac{v^{2}+w^{2}}{u_{0}^{2}}}-1 \\
\Delta \bar{u}=\Delta u-\Delta u^{*}
\end{gathered}
$$

The "small perturbation" assumption considers that the variations of total pressure $\Delta P_{t}$, total temperature $\Delta T_{t}$, and axial velocity $\Delta u$ are small. Moreover, the velocity perturbation $\Delta u$ is decomposed into an isentropic component $\Delta u^{*}$ and a nonisentropic part $\Delta \bar{u}$. The latter is related to the viscous and wave losses (which are sources of entropy; that is why it is called nonisentropic). Note that $\Delta \bar{u}$ is null outside the wake of a body.

For two-dimensional (2-D) applications, the profile drag is the total drag acting upon a body. For three-dimensional (3-D) cases, the vortex drag must also be considered:

$$
\begin{aligned}
D_{\text {vortex }} & =\int_{S_{\text {out }}} \frac{\rho}{2}\left(v^{2}+w^{2}\right) \mathrm{d} S \\
D_{\text {total }} & =D_{\text {profile }}+D_{\text {vortex }}
\end{aligned}
$$

Throughout this paper, the survey plane position used for the evaluation of the far-field drag values will be placed at one chord downstream of the airfoil or wing.

\section{Exergy Method}

Exergy is a classical thermodynamic concept based on the first and second laws of thermodynamics [22,23]. It decomposes the total energy of a system into two components: the exergy $\varepsilon$ (the useful part of the energy) and the anergy " $\mathcal{A}$ " (its useless part). The exergy concept states that any perturbation of the system (perturbation of speed, pressure, or temperature) has an inherent energetic potential that can be completely converted into work if the perturbation is returned to its original (equilibrium) state by means of a reversible transformation. If this transformation is irreversible, only one part of this work potential will be recovered. This can be expressed as follows:

$$
\varepsilon=\delta h_{t}-T_{s_{0}} \delta s=\delta h_{t}-\mathcal{A}
$$

The Arntz formulation will be used throughout this paper [13]. It provides an exergy-based drag force equation when an unpowered and adiabatic case is considered:

$$
D * u_{0}=\dot{\mathcal{A}}+\dot{\varepsilon}_{m}+\dot{\varepsilon}_{\mathrm{th}}
$$

Each term on the right-hand side represents an equation itself, as indicated as follows. Note that the original equations from Arntz [13] require performing an integral on the entire control volume surface enclosing the body (i.e., $S_{0}+S_{\text {lateral }}+S_{\text {out }}$ in Fig. 1). Here, the equations have been slightly modified in order to limit the integration to the outlet surface only (infinite survey plane downstream of the body):

$$
\begin{aligned}
& \dot{\mathcal{A}}=T_{s_{0}} \int_{\text {Sout }} \rho \delta s(\boldsymbol{V} . \boldsymbol{n}) \mathrm{d} S \\
& \delta s=c_{p} \ln \left(\frac{T_{s}}{T_{s_{0}}}\right)-R \ln \left(\frac{P_{s}}{P_{s_{0}}}\right) \\
& \dot{\varepsilon}_{m}=\underbrace{\int_{\text {Sout }} \frac{1}{2} \rho \delta u^{2}(\boldsymbol{V} \cdot \boldsymbol{n}) \mathrm{d} S}_{\dot{E}_{u}}+\underbrace{\int_{\text {Sout }} \frac{1}{2} \rho\left(v^{2}+w^{2}\right)(\boldsymbol{V} \cdot \boldsymbol{n}) \mathrm{d} S}_{\dot{E}_{v}} \\
& +\underbrace{\int_{\text {Sout }}\left(P_{s}-P_{s_{0}}\right)\left[\left(\boldsymbol{V}-\boldsymbol{V}_{0}\right) \cdot \boldsymbol{n}\right] \mathrm{d} S}_{\dot{E}_{p}} \\
& \dot{\varepsilon}_{\text {th }}=\dot{\varepsilon}_{\text {th }_{\text {temperature }}}+\dot{\varepsilon}_{\text {th }_{\text {pressure }}}
\end{aligned}
$$




$$
\begin{aligned}
\dot{\varepsilon}_{\text {th }_{\text {temperature }}}= & \int_{S_{\text {out }}} \rho c_{v} T_{s}(\boldsymbol{V} \cdot \boldsymbol{n})\left[1-\frac{T_{s_{0}}}{T_{s}} \ln \left(\frac{T_{s}}{T_{s_{0}}}\right)\right] \mathrm{d} S \\
& -\int_{S_{\text {out }}} \rho c_{v} T_{S_{0}}(\boldsymbol{V} \cdot \boldsymbol{n}) \mathrm{d} S \\
\dot{\varepsilon}_{\mathrm{th}_{\text {pressur }}}=\int_{S_{\text {out }}} P_{S_{0}} & {\left[1-\frac{\rho}{\rho_{0}} \ln \left(\frac{\rho_{0}}{\rho}\right)\right](\boldsymbol{V} \cdot \boldsymbol{n}) \mathrm{d} S-\int_{S_{\text {out }}} \rho R T_{S_{0}}(\boldsymbol{V} \cdot \boldsymbol{n}) \mathrm{d} S }
\end{aligned}
$$

The total anergy $\dot{\mathcal{A}}$ represents the total amount of exergy that has been already lost by the system (quantified by the entropy increase $\delta s)$. That is why the anergy is also called "exergy destruction" in the fluid dynamics domain [12]. The mechanical exergy outflow rate $\dot{\varepsilon}_{m}$ represents the amount of mechanical power that can be recovered by a so-called exergy recovery system [e.g., boundary-layer ingestion (BLI)]. It is related to the axial and transverse velocity perturbations ( $\dot{E}_{u}$ and $\dot{E}_{v}$, respectively) and the pressure perturbations $\dot{E}_{p}$ ). The thermal exergy outflow rate $\dot{\varepsilon}_{\text {th }}$ represents the amount of thermal power that can be recovered [this is decomposed into its pure thermal and compressible parts in Eq. (15)]. If the exergies are not valued (recovered), they will be gradually destroyed downstream, becoming a loss (i.e., converted into anergy). Also note that the Arntz formulation requires performing integrals in an infinite survey plane, which prevents its use for wind-tunnel data analysis.

As it has been previously shown by Aguirre et al. [16], the transverse exergy can be reexpressed as a function of wake parameters as follows:

$$
\dot{E}_{v \mathrm{wake}}=\int_{S_{w}} \frac{1}{2} \rho \psi \xi u^{*} \mathrm{~d} S
$$

where the axial vorticity $\xi$ " and the isentropic velocity " $u$ "” are given by

$$
\begin{gathered}
\xi=\frac{\partial w}{\partial y}-\frac{\partial v}{\partial z} \\
u^{*}=u_{0} \sqrt{1-\frac{2}{(\gamma-1) M_{o}^{2}}\left[\left(\frac{P_{t}}{P_{t_{0}}}\right)^{(\gamma-1) / \gamma} \zeta-1\right]-\frac{\psi \xi}{u_{0}^{2}}} \\
\zeta=1+\frac{\gamma-1}{2} M_{0}^{2}\left(1-\frac{u^{2}+\psi \xi}{u_{0}^{2}} \frac{T_{t_{0}}}{T_{t}}\right)
\end{gathered}
$$

whereas the stream function $\psi$ is obtained by solving the following Poisson equation:

$$
\frac{d^{2} \psi}{d y^{2}}+\frac{d^{2} \psi}{d z^{2}}=-\xi
$$

Equation (18) states that the transverse exergy can be obtained from the axial vorticity distribution at the survey plane; thus, its integral is now reduced to the wake region (instead of the infinite surface integral required by $\dot{E}_{v}$ in the Arntz formulation).

On the other hand, the drag, exergy, and anergy values will be nondimensionalized as follows:

$$
\begin{aligned}
& C_{D}=\frac{D}{(1 / 2) \rho_{0} u_{0}^{2} S_{\text {ref }}} \\
& \\
& \longrightarrow \text { Velocity component } \\
& \longrightarrow \text { Isentropic part }
\end{aligned}
$$

$$
\begin{aligned}
C_{D_{\varepsilon}} & =\frac{\dot{\varepsilon}_{m}+\dot{\varepsilon}_{\mathrm{th}}+\dot{\mathcal{A}}}{(1 / 2) \rho_{0} u_{0}^{3} S_{\mathrm{ref}}} \\
C_{\dot{\varepsilon}} & =\frac{\dot{\varepsilon}}{(1 / 2) \rho_{0} u_{0}^{3} S_{\mathrm{ref}}} \\
C_{\dot{\mathcal{A}}} & =\frac{\dot{\mathcal{A}}}{(1 / 2) \rho_{0} u_{0}^{3} S_{\mathrm{ref}}}
\end{aligned}
$$

The drag coefficient values are presented in drag counts (dc), defined as $1 / 10,000$ th of $C_{D}\left(1 d c=0.0001 C_{D}\right)$. The exergy-based drag coefficient will be displayed in "power counts" (pc), defined as $1 / 10,000$ th of " $C_{D_{\varepsilon}}$ ", i.e., $1 p c=0.0001 C_{D_{\varepsilon}}$ (the same applies for the exergy/anergy coefficients). Since the exergy-based drag coefficient is equivalent to the force-based drag coefficient, the power counts and drag counts units will be used interchangeably throughout this paper.

\section{A. Limitations of the Meheut Method}

\section{New Velocity Decomposition Method}

At a glance, the velocity decomposition method proposed by Meheut [7] could be used to decompose the exergy equations into its isentropic and nonisentropic components. However, it does not provide a proper decomposition (this is demonstrated later in Fig. 9). To understand why this method is not well suited for the exergy formulation, Eq. (6) must be analyzed. In this equation; the $\Delta u^{*}$ parameter represents the axial velocity perturbation associated with the isentropic development of the transverse flowfield. [Note that the small perturbation assumption is used by Meheut in order to insert this expression into the momentum conservation equation to obtain the drag expression of Eq. (2); however, this isentropic relation is still valid for the entire flowfield, including zones of large perturbations.] Equation (6) enables decomposing the real $u$-velocity component into isentropic $u^{*}$ ) and nonisentropic $\bar{u}$ ) components, where the nonisentropic component is the part of the velocity that appears as a consequence of irreversibilities, i.e., viscous and wave losses.

Nevertheless, the computation of the $\Delta u^{*}$ parameter relies on the measurement of the $y$ - and $z$-velocity components, assuming an isentropic relation. This means that the Meheut's decomposition assumes that the $v$ and $w$ velocities are isentropic in the entire domain (i.e., inside and outside the wake). This is true outside of the viscous wake of a body, where the flow is isentropic and then the $u, v$, and $w$ components are truly isentropic. However, inside the wake and the boundary layer, all those three velocities have an isentropic and a nonisentropic component as shown in Fig. 2.

Thus, using Eq. (6) to calculate the isentropic $u$ velocity from the real $v$ and $w$ velocities (that contain both the isentropic and the nonisentropic components) is not exact and will lead to a small error. This error has shown to be negligible for far-field method applications. However, using this approach in the exergy equations leads to major errors. Thus, a new velocity decomposition method suited for the exergy analysis is proposed as follows.

\section{B. Proposed Velocity Decomposition Method}

The starting point is the isentropic relation used by Meheut [7]:

$$
\frac{P_{s}^{*}}{P_{s_{0}}}=\left[1+\frac{\gamma-1}{2} M_{0}^{2}\left(1-\frac{u^{* 2}+v^{2}+w^{2}}{u_{0}^{2}}\right)\right]^{\gamma /(\gamma-1)}
$$


Here, the $v$ and $w$ velocities contain both the isentropic and nonisentropic components. Thus, the same equation combines both purely isentropic with nonisentropic components, which leads to an inaccuracy. However, this equations turns out to be exact if the isentropic velocity is used instead:

$$
\frac{P_{s}^{*}}{P_{s_{0}}}=\left[1+\frac{\gamma-1}{2} M_{0}^{2}\left(1-\frac{V^{* 2}}{u_{0}^{2}}\right)\right]^{\gamma /(\gamma-1)}
$$

This equation simply relates the velocity magnitude with the static pressure in a fully isentropic field. It can be rewritten as

$$
\frac{P_{s}^{*}}{P_{s_{0}}}=\left[1+\frac{\gamma-1}{2} M_{0}^{2}\left(1-\frac{u^{* 2}+v^{* 2}+w^{* 2}}{u_{0}^{2}}\right)\right]^{\gamma /(\gamma-1)}
$$

Note that Eq. (29) is similar to Eq. (27), with the exception that the isentropic $v$ and $\bar{w}$ velocities are now used. In fact, Meheut [7] was only concerned with the isentropic $u$ velocity because his drag formulation [Eq. (2)] only deals with this velocity component. This was well suited for far-field drag prediction but not for the exergy method. that

Interestingly, Eq. (28) still holds for a real flow by acknowledging

$$
P_{s} \approx P_{s}^{*}
$$

This means that the actual static pressure and the isentropic static pressure are practically equals in the entire domain. In other words, the static pressure field is not affected inside the viscous regions. This fact was demonstrated by Meheut [7], and it will be shown later in Fig. 7. Hence, Eq. (28) can be rewritten as

$$
\frac{P_{s}}{P_{s_{0}}}=\left[1+\frac{\gamma-1}{2} M_{0}^{2}\left(1-\frac{V^{* 2}}{u_{0}^{2}}\right)\right]^{\gamma /(\gamma-1)}
$$

This enables expressing the isentropic velocity magnitude at each point of the domain as a direct function of the local static pressure, regardless of the region analyzed (i.e., isentropic or nonisentropic):

$$
V^{*}=u_{0} \sqrt{1-\frac{2}{(\gamma-1) M_{0}^{2}}\left[\left(\frac{P_{s}}{P_{s_{0}}}\right)^{(\gamma-1) / \gamma}-1\right]}
$$

Since the static pressure varies across the entire domain, it will be more convenient to express the previous equation in terms of the total pressure because this parameter only changes inside the boundary layer and the wake of a body. The static pressure can be related to the total pressure by using isentropic relations [7]:

$$
\frac{P_{s}}{P_{s_{0}}}=\frac{P_{t}}{P_{t_{0}}} * \zeta^{\frac{\gamma}{(\gamma-1)}}
$$

where

$$
\zeta=1+\frac{\gamma-1}{2} M_{0}^{2}\left(1-\frac{V^{2}}{u_{0}^{2}} \frac{T_{t_{0}}}{T_{t}}\right)
$$

Then, Eq. (32) becomes

$$
V^{*}=u_{0} \sqrt{1-\frac{2}{(\gamma-1) M_{0}^{2}}\left[\left(\frac{P_{t}}{P_{t_{0}}}\right)^{(\gamma-1) / \gamma} * \zeta-1\right]}
$$

This gives the isentropic velocity magnitude at any point of the domain as a function of the local total pressure, total temperature, and real velocity (contained inside the $\zeta$ term).

To obtain the components of the isentropic velocity, a major assumption will be made: the isentropic velocity vector is locally aligned with the real velocity vector. This assumption is based on the fact that the losses are convected by the flow; hence, the nonisentropic velocity vector will be aligned with the real velocity vector, and consequently the isentropic velocity vector will also be aligned. Thus, the direction cosines of the real velocity vector will be used to obtain the isentropic velocity components along the $x, y$, and $z$ axes (see Fig. 3):

$$
\left\{\begin{array}{c}
\theta=\operatorname{arctg}\left(\frac{w}{\sqrt{u^{2}+v^{2}}}\right) \\
\alpha=\operatorname{arctg}\left(\frac{w}{u}\right) \\
\beta=\operatorname{arctg}\left(\frac{v}{u}\right)
\end{array}\right.
$$

Then, the isentropic (inviscid) velocity components become

$$
\begin{gathered}
u^{*}=V^{*} \cos (\theta) \cos (\beta) \\
v^{*}=V^{*} \cos (\theta) \sin (\beta) \\
w^{*}=V^{*} \sin (\theta)
\end{gathered}
$$

Moreover, the nonisentropic (viscous) velocity components can be obtained by subtracting the isentropic velocity field from the real velocity field:

$$
\begin{aligned}
& \bar{u}=u-u^{*} \\
& \bar{v}=v-v^{*} \\
& \bar{w}=w-w^{*}
\end{aligned}
$$

\section{Exergy Breakdown Method}

The decomposition of the velocity field into its isentropic and nonisentropic components leads to the decomposition of the exergy terms into its isentropic and nonisentropic components. This exergy breakdown intends to highlights the physical origin of each type of exergy. "Isentropic exergy" stands for the exergy that is available at some point of the domain and that was created by a pure isentropic process (e.g., the velocity perturbation field outside the boundary layer). A "nonisentropic exergy" stands for the exergy available at some point of the domain and that was created by an entropygenerating process (e.g., the velocity deficit inside the boundary layer or wake).

The interest of decomposing each term of the exergy equation into its isentropic and nonisentropic parst is related to the fact that the isentropic exergy is not useful from an engineering point of view for 2-D cases because it represents a self-recovered exergy [15]: the perturbed flow outside the wake will follow an isentropic process

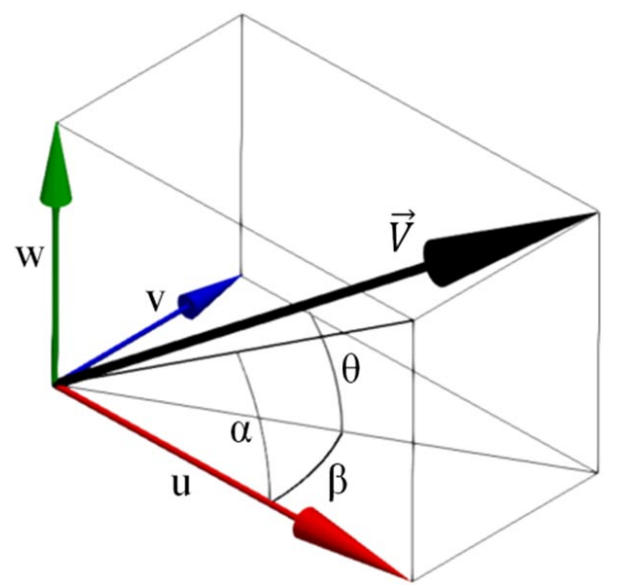

Fig. 3 Real velocity vector components. 
downstream, thereby recovering all its related exergy potential once it reaches the equilibrium condition. On the other hand, the nonisentropic exergy represents the part of the exergy available inside an entropy-generating region. Hence, if this exergy is not recovered, it will be destroyed downstream by entropy-generating processes (e.g., turbulent mixing): it is up to the designer to recover that exergy before it becomes anergy. Hence, the exergy breakdown can provide a refined tool for practical design purposes. This concept will be further discussed in the next sections.

\section{A. Mechanical Exergy Breakdown}

The isentropic exergies are obtained by replacing their actual velocity components by the isentropic ones:

$$
\begin{gathered}
\dot{E}_{u}^{*}=\int_{S_{\text {out }}} \frac{1}{2} \rho\left(u^{*}-u_{0}\right)^{2} u^{*} \mathrm{~d} S \\
\dot{E}_{v}^{*}=\int_{S_{\text {out }}} \frac{1}{2} \rho\left(v^{* 2}+w^{* 2}\right) u^{*} \mathrm{~d} S \\
\dot{E}_{p}^{*}=\int_{S_{\text {out }}}\left(P_{S}-P_{s_{0}}\right)\left(u^{*}-u_{0}\right) \mathrm{d} S
\end{gathered}
$$

As a reminder, a survey plane normal to the upstream flow direction is considered here. (That is why the $\boldsymbol{V} \cdot \boldsymbol{n}$ term becomes $u^{*}$.) On the other hand, the nonisentropic components are obtained by subtracting the isentropic field from the real field:

$$
\begin{aligned}
& \overline{\dot{E}_{u}}=\dot{E}_{u}-\dot{E}_{u}^{*} \\
& \overline{\dot{E}_{v}}=\dot{E}_{v}-\dot{E}_{v}^{*} \\
& \overline{\dot{E}_{p}}=\dot{E}_{p}-\dot{E}_{p}^{*}
\end{aligned}
$$

The same decomposition also applies for the mechanical exergy:

$$
\begin{gathered}
\dot{\varepsilon}_{m}^{*}=\dot{E}_{u}^{*}+\dot{E}_{v}^{*}+\dot{E}_{p}^{*} \\
\bar{\varepsilon}_{m}=\dot{\varepsilon}_{m}-\dot{\varepsilon}_{m}^{*}
\end{gathered}
$$

It is interesting to note that $\dot{\varepsilon}_{m}$ and $\dot{\varepsilon}_{m}^{*}$ require an infinite surface integral on the entire survey plane; however, $\overline{\dot{\varepsilon}_{m}}$ only requires a wake integral. This is because the difference between the real field and the isentropic field (i.e., $\dot{\varepsilon}_{m}-\dot{\varepsilon}_{m}^{*}$ ) will provide the nonisentropic component (i.e., $\overline{\dot{\varepsilon}_{m}}$ ) that is only related to the viscous and wave losses; thus, it is confined to the boundary layer, shock wave, and wake region. Hence, a surface integral limited to the wake region is sufficient to compute $\overline{\dot{\varepsilon}_{m}}$ properly, simply because it has a null value at any point outside the boundary layer and wake zones. (This is because $\overline{\dot{\varepsilon}_{m}}$ is a function of the nonisentropic velocity, which is limited to the wake region.) The same reasoning also applies for its components: $\bar{E}_{u}, \bar{E}_{v}$, and $\overline{\dot{E}_{p}}$.

\section{B. Thermal Exergy Breakdown}

Thermal exergy also deals with velocity components; thus, it admits a decomposition into its isentropic and nonisentropic parts. However, the thermal exergy also contains other parameters that are affected by the viscous effects inside the boundary layer and the wake: the static temperature and density fields. Thus, they must be decomposed beforehand by using the isentropic relations applied to Eq. (28) as follows:

$$
T_{s}^{*}=T_{s_{0}}\left[1+\frac{\gamma-1}{2} M_{0}^{2}\left(1-\frac{V^{* 2}}{u_{0}^{2}}\right)\right]
$$

$$
\rho^{*}=\rho_{0}\left[1+\frac{\gamma-1}{2} M_{0}^{2}\left(1-\frac{V^{* 2}}{u_{0}^{2}}\right)\right]^{1 /(\gamma-1)}
$$

Then, by using Eqs. (151) and (22), and considering a survey plane perpendicular to the upstream flow direction (i.e., $\boldsymbol{V} \cdot \boldsymbol{n}=u$ ), the isentropic thermal exergy [Eq. (15)] becomes

$$
\begin{gathered}
\dot{\varepsilon}_{\mathrm{th}}^{*}=\dot{\varepsilon}_{\mathrm{th}_{\text {temperature }}^{*}}+\dot{\varepsilon}_{\mathrm{th}_{\text {pressure }}^{*}} \\
\dot{\varepsilon}_{\mathrm{th}_{\text {temperature }}}^{*}=\int_{S_{\text {out }}} \rho^{*} c_{v} T_{s}^{*} u^{*}\left[1-\frac{T_{S_{0}}}{T_{s}^{*}} \ln \left(\frac{T_{s}^{*}}{T_{s_{0}}}\right)\right] \mathrm{d} S-\int_{S_{\text {out }}} \rho^{*} c_{v} T_{s_{0}} u^{*} \mathrm{~d} S \\
\dot{\varepsilon}_{\mathrm{th}_{\text {pressure }}}^{*}=\int_{S_{\text {out }}} P_{S_{0}}\left[1-\frac{\rho^{*}}{\rho_{0}} \ln \left(\frac{\rho_{0}}{\rho^{*}}\right)\right] u^{*} \mathrm{~d} S-\int_{S_{\text {out }}} \rho^{*} R T_{s_{0}} u^{*} \mathrm{~d} S
\end{gathered}
$$

Finally, the nonisentropic thermal exergy is given by subtracting the isentropic part from the total field:

$$
\overline{\dot{\varepsilon}_{\mathrm{th}}}=\dot{\varepsilon}_{\mathrm{th}}-\dot{\varepsilon}_{\mathrm{th}}^{*}
$$

As was explained before, the integral of the nonisentropic thermal exergy is also reduced to the wake region.

\section{Exergy-Based Drag Breakdown}

According to the Arntz proposition [Eq. (24)], the drag field distribution at a given survey plane position is a combination of the exergy and anergy fields. Since the exergy-based drag is a function of the exergy and, in turn, the exergy can be broken down into its isentropic and nonisentropic parts, we can extend this concept in order to decompose the exergy-based drag field into its isentropic and nonisentropic parts as follows:

$$
\begin{gathered}
C_{D_{\varepsilon}}^{*}=\frac{\dot{\varepsilon}_{m}^{*}+\dot{\varepsilon}_{\mathrm{th}}^{*}}{(1 / 2) \rho_{0} u_{0}^{3} S_{\mathrm{ref}}} \\
C_{D_{\varepsilon}}=C_{D_{\varepsilon}}-C_{D_{\varepsilon}}^{*}
\end{gathered}
$$

Again, the objective of this breakdown is to highlight the physical origin of the drag components at a given survey plane position. "Isentropic drag" stands for the part of the drag field distribution linked to perturbations of isentropic origin (e.g., the velocity perturbation outside the boundary layer or wake). On the other hand, "nonisentropic drag" is the part of the drag field distribution linked to perturbations created by entropy-generating mechanisms (e.g., the velocity deficit inside the boundary layer or wake). Of course, this is plane dependent in the sense that a particle could have followed a purely isentropic process up to certain plane position; but, maybe downstream, this particle penetrates the wake. At that point, the particle will contain both isentropic and nonisentropic perturbations.

Note that the isentropic drag coefficient $C_{D_{\varepsilon}}^{*}$ does not include the anergy term as it does in the total drag coefficient equation [Eq. (24)]. This is because the anergy takes into account the losses and it must not be considered for the isentropic drag component. Nevertheless, anergy is implicitly present in Eq. (58) across the $C_{D_{\varepsilon}}$ term; thus, the anergy is included into the nonisentropic drag coefficient.

On the other hand, $C_{D_{\varepsilon}}$ and $C_{D_{\varepsilon}}^{*}$ must be integrated on the entire survey plane since these fields varies across the entire domain. However, $C_{\overline{D_{\varepsilon}}}$ only requires a wake integral because its field is zero outside the wake region:

$$
\int_{S_{\text {out }}} C_{D_{\varepsilon}} \mathrm{d} S=\int_{S_{\text {out }}} C_{D_{\varepsilon}}^{*} \mathrm{~d} S+\int_{S_{w}} C_{\bar{D}_{\varepsilon}} \mathrm{d} S
$$

For 2-D cases, the integral of the nonisentropic exergy drag coefficient $C_{\overline{D_{\varepsilon}}}$ will provide the profile drag. Moreover, the integral 
of the isentropic component is negligible, as will be shown later in Fig. 26:

For $\overline{2-\mathrm{D}}$ cases,

$$
\int_{S_{\text {out }}} C_{D_{\varepsilon}} \mathrm{d} S=\int_{S_{\text {out }}} C_{D_{\varepsilon}}^{*} \mathrm{~d} S+\int_{S_{w}} C_{\bar{D}_{\varepsilon}} \mathrm{d} S=0+\text { profile drag }
$$

For 3-D cases, $C_{\bar{D}_{\varepsilon}}$ will also provide the profile drag. The induced drag is automatically transferred to the isentropic component $C_{D_{\varepsilon}}^{*}$ when the velocity decomposition is used. Hence, the total drag becomes the following:

For 3-D cases,

$$
\begin{aligned}
\int_{S_{\text {out }}} C_{D_{\varepsilon}} \mathrm{d} S & =\int_{S_{\text {out }}} C_{D_{\varepsilon}}^{*} \mathrm{~d} S+\int_{S_{w}} C_{\bar{D}_{\varepsilon}} \mathrm{d} S \\
& =\text { induced drag }+ \text { profile drag }
\end{aligned}
$$

This mathematical behavior is not surprising because the same happened with the far-field formulations [7] where the momentum conservation equation provided the total drag, whereas Meheut's method (that uses the velocity decomposition) only provided the profile drag for 2-D and 3-D cases. In fact, the transverse flow associated with the wingtip vortices is purely isentropic outside the wake; thus, it is already included in the $C_{D_{\varepsilon}}^{*}$ term through the $\dot{\varepsilon}_{m}^{*}$ component. This explains why $D_{\overline{D_{\varepsilon}}}$ does not contain the induced drag. However, this transverse flow is linked to the axial vorticity field left downstream by the body inside its wake [16], which is accounted for separately by the $\dot{E}_{v \text { wake }}$ method [Eq. (18)]. Thus, in the far-field and exergy methods with velocity decomposition, the induced drag (transverse exergy) must be added to the profile drag (nonisentropic exergy drag). This will be explained in detail in the next sections.

\section{CFD Data}

Two-dimensional and 3-D CFD data will be used for the analysis of the velocity decomposition method and the new exergy formulations. The 2-D case is a NACA 0012 airfoil with a sharp trailing edge. The $3-\mathrm{D}$ case is a rectangular wing with an aspect ratio of eight and with the same airfoil and a rounded wingtip. In both cases a C-block structured grid with wake refinement was used (see Fig. 4). The domain extent is 150 chords in all directions for the 2-D case and 30 chords for the 3-D case. For the 2-D case, a grid refinement was performed and the near-field drag value compared against experimental data of the bibliography [24-27], as shown in Fig. 5. Then, the mesh of 593,000 cells was selected for the 2-D case, which ensured the correct capture of all the physical phenomena even in transonic conditions. For the 3-D case, a similar 2-D mesh was extruded spanwise, resulting in a mesh of 9.2 million cells. The mesh blocking and refinement on the wake region were different for each angle of attack for both 2-D and 3-D meshes: the refinement zone follows the wake deviation in order to ensure a proper capture of the wake.

The 2-D and 3-D cases were analyzed for several angles of attack at constant Mach numbers of 0.3 and 0.8 , at a Reynolds number of

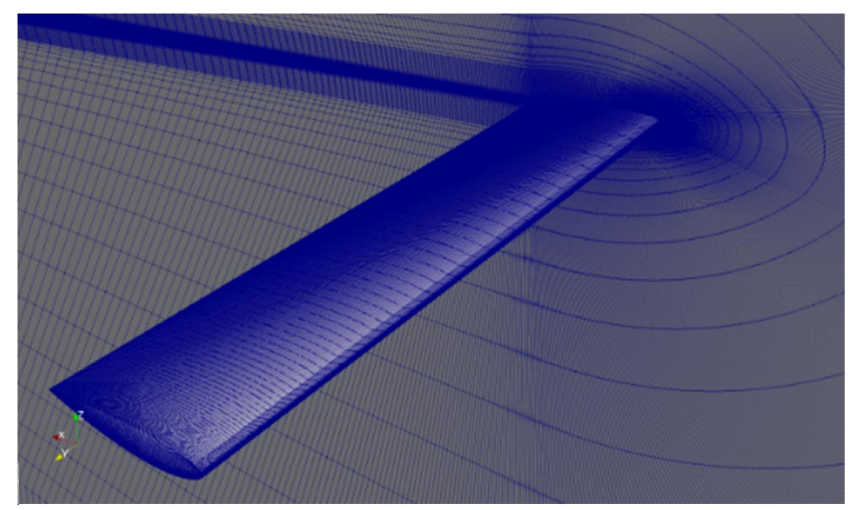

Fig. 4 3-D mesh for $\alpha=0 \mathrm{deg}$.

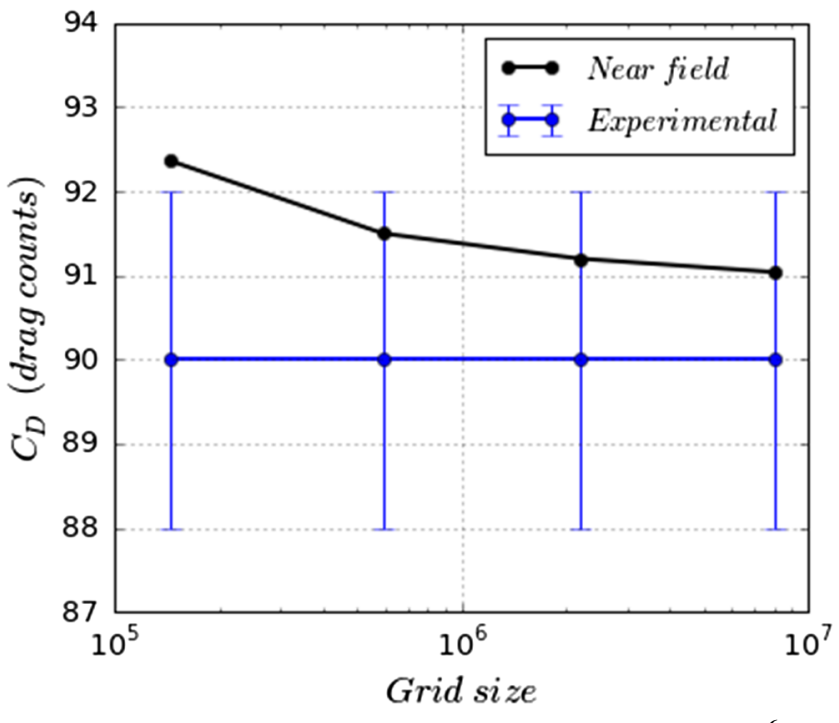

Fig. 5 Grid convergence for $\alpha=0 \mathrm{deg}, M=0.3$, and $R e=3.10^{6}(2-\mathrm{D}$ case).

$3 \times 10^{6}$. In all the cases, Reynolds-averaged Navier-Stokes simulations were performed with the Spalart-Allmaras turbulence model. A first quick convergence was done with a first-order discretization (flow and turbulence) by about 3000 iterations, followed by a final second-order discretization convergence as shown in Fig. 6 . All the simulations were left running until the near-field drag coefficient residual was less than 0.1 drag counts. At the same time, the residuals must reach their maximum precision in order to ensure that the airfoil's losses were completely transmitted (convected) downstream. Then, the $y^{+}$parameter was controlled in order to verify that $y^{+} \leq 1$ everywhere around the body (as required by the Spalart-Allmaras model). The resulting CFD data were analyzed with a ParaView plugin called Epsilon [19]: an open-source code developed by the National Higher French Institute of Aeronautics and Space (ISAESUPAERO), which performs far-field and exergetic analyses.

\section{Velocity Decomposition Analysis}

In this section, different flowfield parameters are analyzed in order to highlight the facts already mentioned during the development of the formulation.

\section{A. Static Pressure Field}

The starting point of the velocity decomposition procedure was the acknowledgement that the static pressure field is practically not

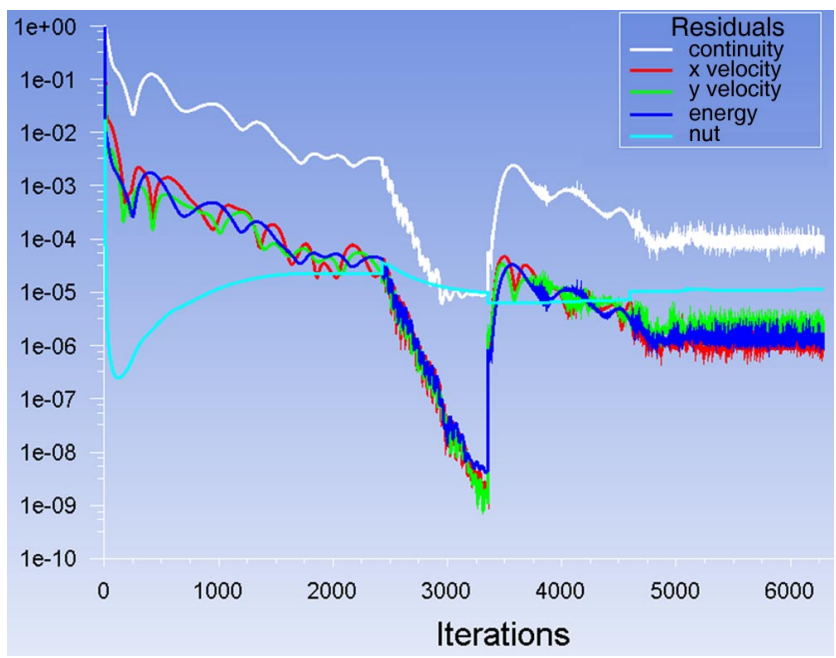

Fig. 6 Residuals convergence for the airfoil at $\alpha=0 \mathrm{deg} / M=0.3$ (NUT = turbulent kinetic viscosity). 


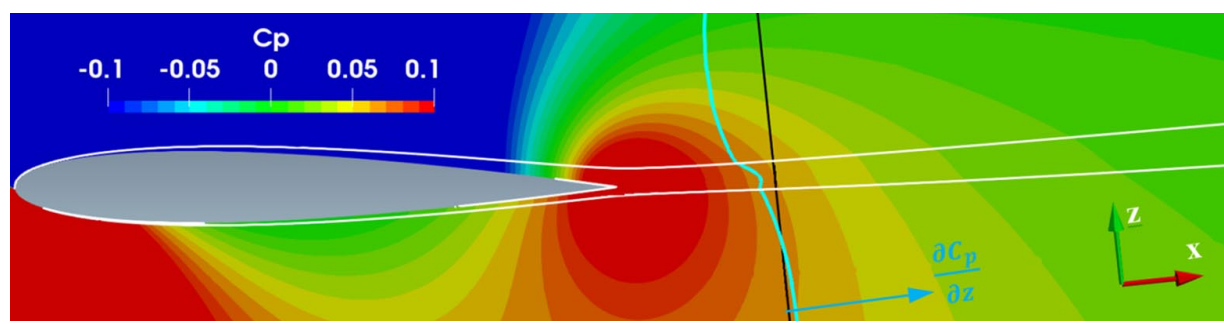

Fig. $7 C_{p}$ field at $\alpha=6 \mathrm{deg} / M=0.3$.

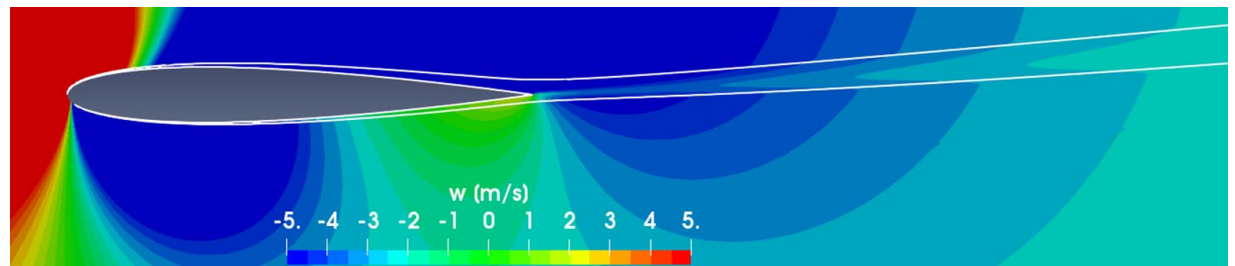

Fig. 8 The $w$-velocity field at $\alpha=6 \mathrm{deg} / M=0.3$.

affected inside the boundary layer and wake regions as it was proven by Meheut [7]. This has led to the assumption that the isentropic static pressure is actually the real static pressure (i.e., $P_{s} \approx P_{s}^{*}$ ). This is observed in Fig. 7, where the pressure coefficient field is shown around a NACA 0012 airfoil, along with a white line that delimits the boundary layer and wake region. The field outside these lines is isentropic and the field inside is nonisentropic. It can be clearly seen that the $C_{p}$ field does not suffer a distortion inside the nonisentropic region. Instead, the $C_{p}$ contour lines cross the viscous region without suffering a noticeable kink in those lines. To highlight this fact, Fig. 7 also shows the distribution of the $C_{p}$ gradient along a black survey line (placed normal to the upstream flow direction). The $C_{p}$ gradient is a parameter very sensitive to the distortions. However, it is observed that the degree of distortion suffered by this gradient distribution is quite small inside the viscous region.

Any kink of the contour lines would be a clear trace of the presence of a nonisentropic region. This can be observed in Fig. $\underline{8}$ for the w-velocity field, where the contour lines are deformed across the wake because of the presence of a nonisentropic region (purely viscous effect in this case). Hence, it can be concluded that the pressure field is practically not affected by the viscous phenomena because their contour lines are not kinked; hence, $P_{s} \approx P_{s}^{*}$.

\section{B. Meheut Velocity Decomposition Applied to the Exergy Method}

If the velocity decomposition proposed by Meheut [7] is used for the breakdown of the exergy equations, it does not succeed in taking away the kink (distortion) from the contour lines across the nonisentropic region. This can be observed for the isentropic axial exergy field $\dot{E}_{u}^{*}$ in Fig. 9, where a large kink is observed across the viscous region (see upper left of upper image). Instead, the new method

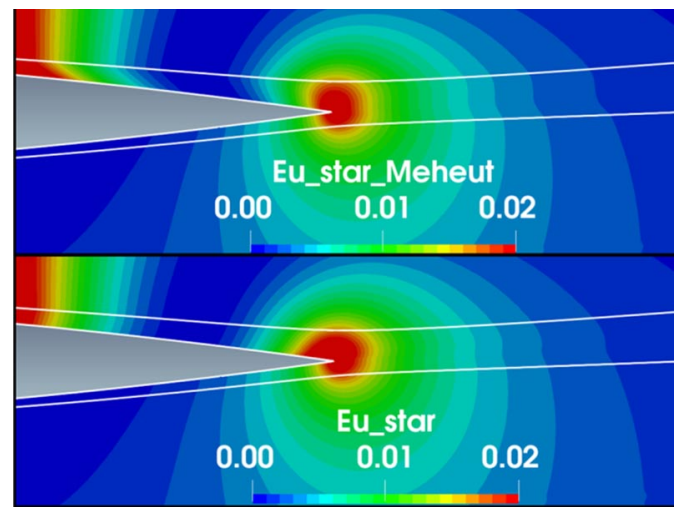

Fig. $9 \dot{E}_{u}^{*}$ field at $\alpha=6 \mathrm{deg} / M=0.3$. (lower image) provides a smoother field than Meheut's method. The kink of the contour line for Meheut's method is even worse for $\dot{E}_{v}^{*}$ because the transverse velocities' components are not decomposed in this method (there are no isentropic $v$ and $w$ velocities). The resulting contour kink of the Meheut method did not compromise the accuracy in far-field method applications. Nevertheless, these distortions are shown to be excessive for the exergy method. In fact, the Meheut profile drag equation [Eq. (2)] only deals with the square of the velocity components, whereas the exergy terms [Eqs. (43), (44), and (49)] deal with the cubic law of the velocity components. Hence, any distortion of the contours of the velocity components across the nonisentropic region will be amplified by the exergy equations, leading to unacceptable errors.

\section{New Velocity Decomposition}

The new velocity decomposition formulation enables extracting the isentropic part " $u$ " " and the nonisentropic part " $\bar{u}$ " from the velocity field $u$. This is shown in Fig. 10, where the actual velocity field is shown as the starting point. Then, in Fig. 11, only the isentropic part is retained; i.e., the effect of the viscous losses on the velocity field is taken away, leaving a pure inviscid flowfield. Note that this field is equivalent to the potential flow around the airfoil [17]. On the other hand, Fig. 12 shows the nonisentropic part that has been extracted; i.e., it only contains the viscous effect on the velocity field. Since the boundary layer and the wake create losses, and those

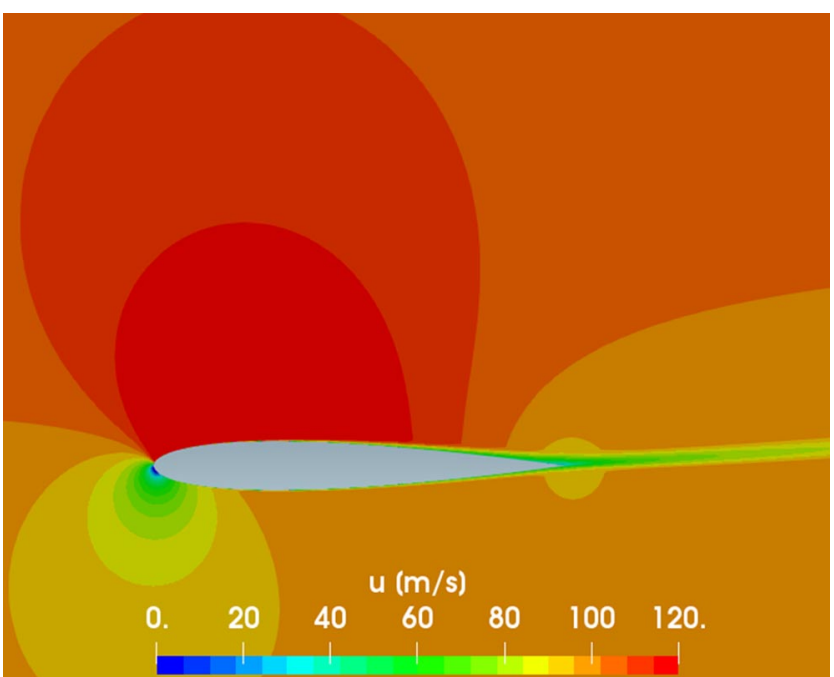

Fig. 10 The $u$-velocity field at $\alpha=6 \mathrm{deg} / M=0.3$. 


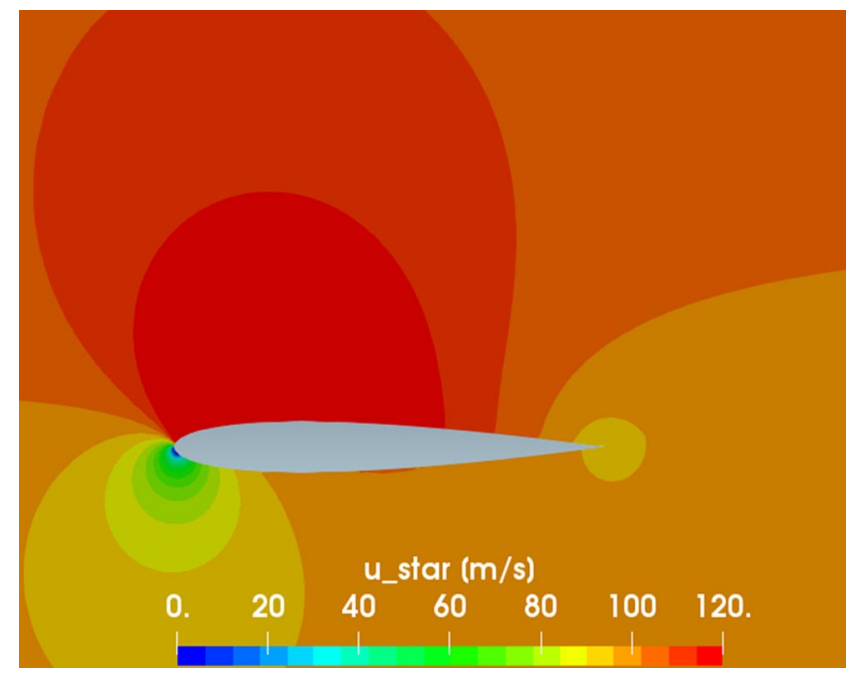

Fig. 11 Isentropic velocity field $u^{*}$ at $\alpha=6 \mathrm{deg} / M=0.3$.

losses are observed as a velocity deficit, the nonisentropic velocity field is negative inside the viscous region. This velocity deficit is due to the loss of momentum inside the viscous region, associated with the drag (momentum transfer from the fluid to the body). Also note that this nonisentropic velocity is null outside the boundary layer and wake region. That is why its integral can be reduced to the wake region only.

To analyze the decomposition capabilities of the proposed method, a close-up is made around the trailing-edge region for the isentropic uand w-velocity fields (Figs. 13 and 14, respectively). The $u^{*}$ field shows a very small kink across the boundary-layer limit and a w-like kink pattern across the wake region. Nevertheless, this kink is very small.

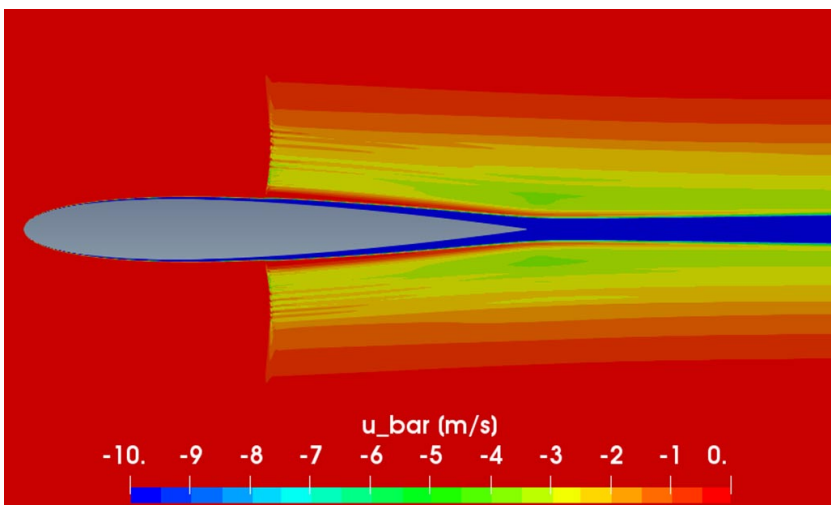

Fig. 15 Nonisentropic velocity field $\bar{u}$ at $\alpha=0 \mathrm{deg} / M=0.8$.

On the other hand, the $w^{*}$ velocity field shows an s-like kink pattern across the wake. This oscillation is developed around the mean contour line inside the wake region. It can be seen that the horizontal deviations of the kink line (taking the mean line as a reference) are not small. However, it has not shown to have a negative impact on the exergy decomposition. In fact, the positive and negative oscillations (around the mean line) of the contour lines inside the wake seem to be self-compensated during the integration. (This is a matter of further study.) Moreover, the fact of decomposing the transverse velocities represents a significant advantage over the Meheut method, where the $v$ and $w$ velocities are not decomposed at all.

It is important to highlight that the nonisentropic velocity field is related not only to the viscous losses but also to the wave losses. This can be clearly seen in Fig. 15 where a velocity deficit is also observed across the shock wave region.

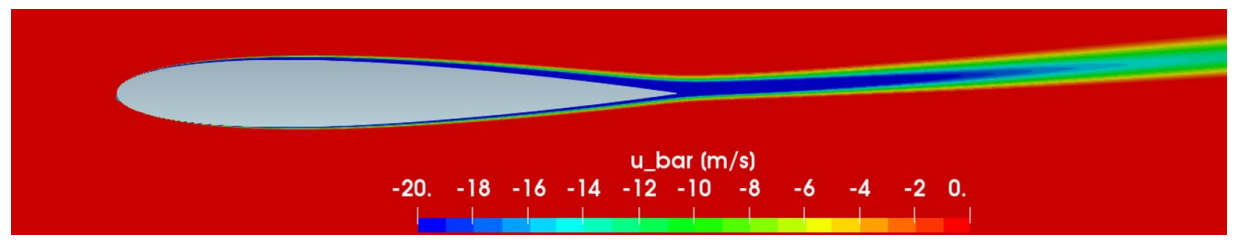

Fig. 12 Nonisentropic velocity field $\bar{u}$ at $\alpha=6 \mathrm{deg} / M=0.3$.

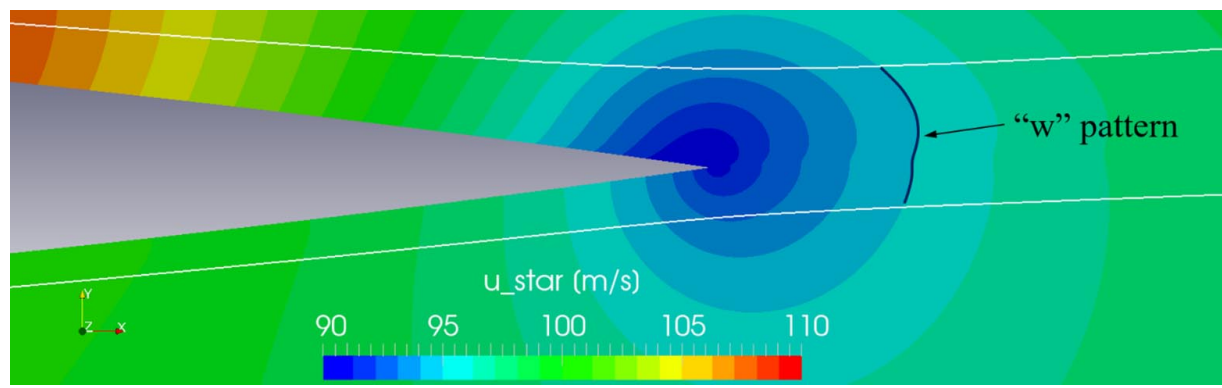

Fig. 13 Isentropic velocity field $u^{*}$ at $\alpha=6 \mathrm{deg} / M=0.3$.

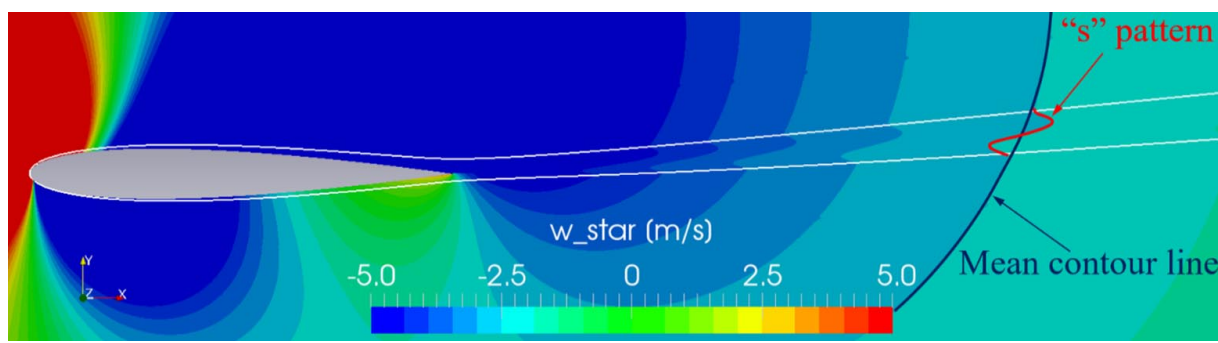

Fig. 14 Isentropic velocity field $w^{*}$ at $\alpha=6 \mathrm{deg} / M=0.3$. 


\section{Exergy Breakdown Flowfield Analysis}

In this section, an analysis of the different decomposed exergy parameters will be made in order to highlight the facts already mentioned during the development of the formulation. This will be performed with 2-D and 3-D data, for subsonic and transonic conditions as well.

\section{A. 2-D Subsonic}

The velocity decomposition applied to the exergy formulation enables us to obtain its isentropic and nonisentropic components. This is shown for the case of the exergy-based drag coefficient in Figs. 16-18. Here, the reader is reminded that the breakdown of any parameter into its isentropic and nonisentropic components intends to highlight the physical origin of each component: the isentropic part is related to isentropic perturbations, whereas the nonisentropic part is related to perturbations created by entropy-generating mechanisms.

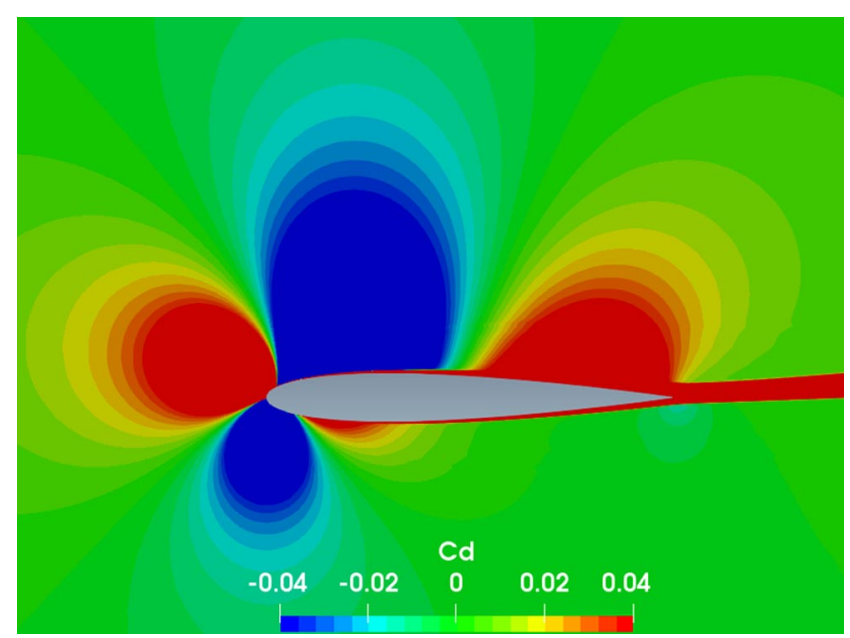

Fig. 16 Exergy-based drag coefficient field $C_{D_{\varepsilon}}$ at $\alpha=6 \mathrm{deg} / M=0.3$.

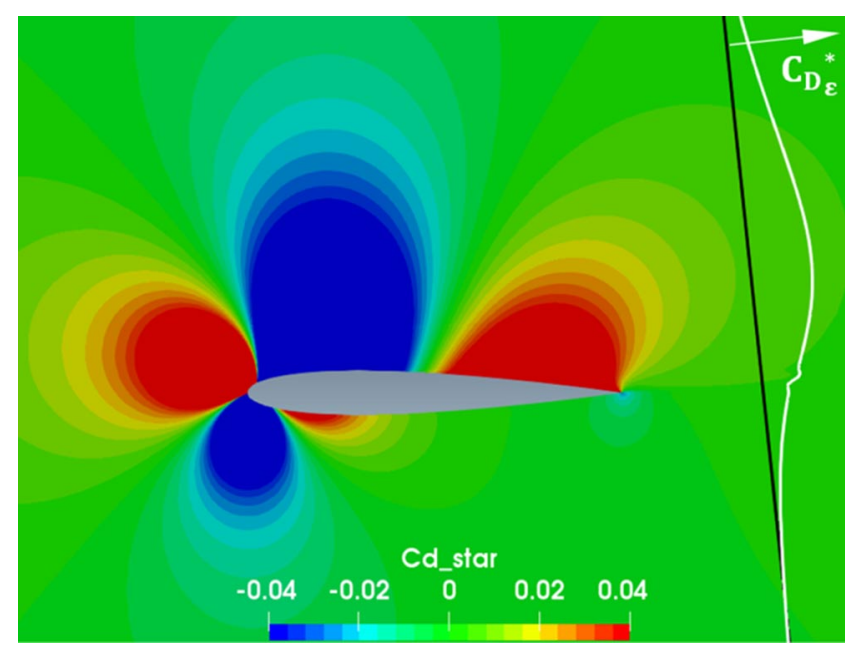

Fig. 17 Isentropic drag coefficient field $C_{D_{\varepsilon}}^{*}$ at $\alpha=6 \mathrm{deg} / M=0.3$.
Note that the isentropic component $C_{D_{\varepsilon}}^{*}$ from Fig. 17 takes away the influence of the losses that occur inside the boundary layer and the wake. It only retains the part of the field associated with an isentropic evolution (equivalent to a potential flow [17]). Also note that this field varies at every point of the domain: a survey plane placed downstream of the airfoil (black line) will detect nonzero values of this field along it (white curve). However, when these values are integrated along the line, the result is a zero net contribution to the drag coefficient in 2-D cases (it will be shown numerically in Fig. 26). This confirms the fact that the isentropic exergy field is not interesting for design purposes in 2-D cases [15] because the related exergy is self-recovered downstream since the flow particles follow an isentropic trajectory; they return to the original (equilibrium) state by following a reversible path. This highlights the need of decomposing the exergy formulations in order to retain only the interesting part of the exergy from a design point of view: the nonisentropic field.

The nonisentropic exergy-based drag coefficient field (related to viscous and shock wave losses) is shown in Fig. 18. This field is zero outside the boundary layer and the wake, as expected, since no entropy-generating mechanism exists there. This can be best observed by the help of the black survey line placed normal to the upstream flow direction and along which the $C_{\overline{D_{\varepsilon}}}$ distribution is shown. The integral of this distribution [Eq. (58)] will provide the profile drag coefficient; since this field is null outside the wake, the related integral is reduced to the wake region. This demonstrates that the decomposed exergy formulation is suited for wind-tunnel data analysis, whereas the original Arntz method requires an infinite survey plane integral since it also integrates the isentropic components (whose field varies at any point of the domain). Moreover, the $C_{\overline{D_{\varepsilon}}}$ distribution along the survey line matches the distribution of $C_{D_{\varepsilon}}$ (not shown here for simplicity); thus, it will also differ from the Meheut profile drag distribution [15].

To assess the effectiveness of the velocity decomposition for the exergy formulation, the isentropic drag field close to the trailing-edge region is analyzed in Fig. 19. It can be observed that the contour lines only present a very weak kink across the wake region for small angles of attack. For larger angles of attack (Fig. 20), the kink increases but it adopts an s-like pattern that is self-compensated during the integration.

The conclusions from the analysis of the isentropic and nonisentropic exergy drag fields also apply to its components (mechanical exergy, thermal exergy, and so on), and thus are not shown here for simplicity.

\section{B. 2-D Transonic}

In transonic conditions, the nonisentropic exergy-based drag coefficient field also highlights the wave losses as shown in Fig. 21 (because the shock wave is also an entropy-generating mechanism). Its related distribution along the black survey line is also shown, where the wave drag corresponds to the part of the distribution lying on the top of the green region. This wave drag can be extracted from the $C_{\overline{D_{\varepsilon}}}$ distribution by using the method previously proposed by Aguirre and Duplaa [14], which is suited for wind-tunnel testing.

\section{3-D Subsonic}

To analyze the impact of the velocity decomposition method on the exergy equations, a survey plane will be placed at one chord downstream of the wing, normal to the upstream flow direction (Fig. 22).

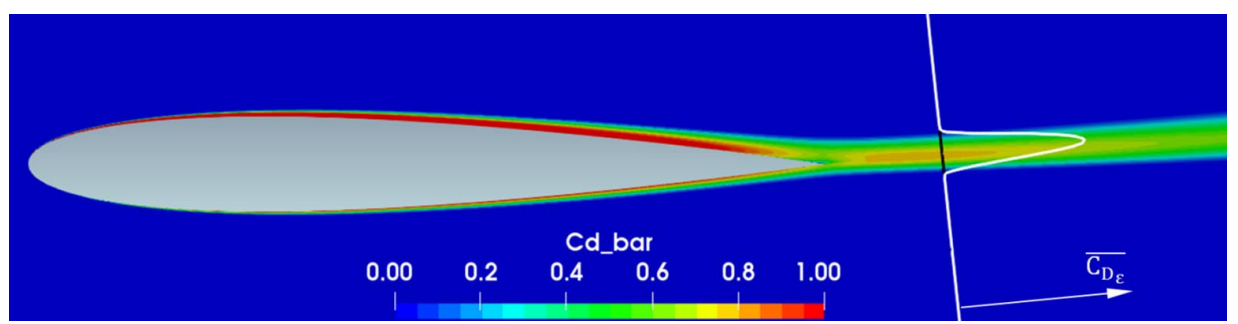

Fig. 18 Nonisentropic drag coefficient field $\overline{C_{D_{\varepsilon}}}$ at $\alpha=6 \mathrm{deg} / M=0.3$. 


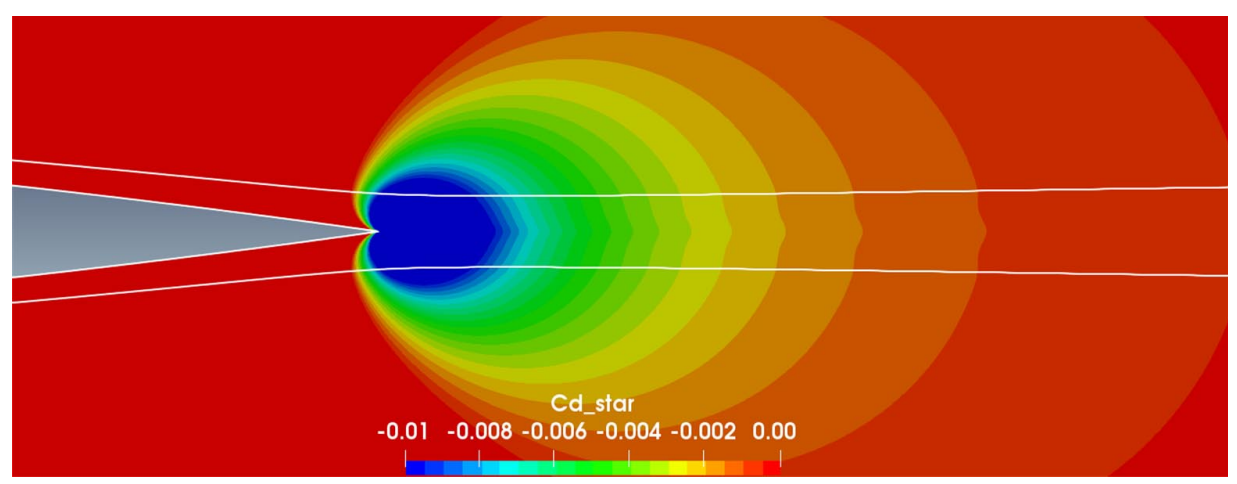

Fig. 19 Isentropic drag coefficient field $C_{D_{\varepsilon}}^{*}$ at $\alpha=0 \mathrm{deg} / M=0.3$.

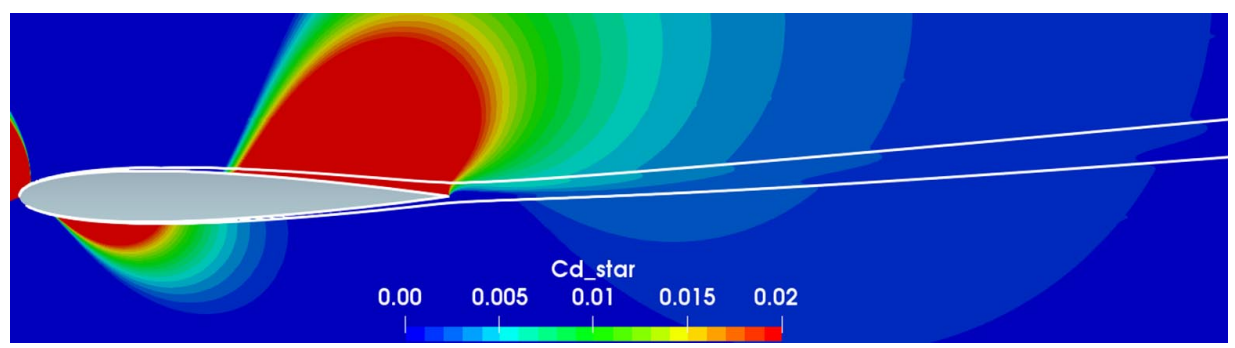

Fig. 20 Isentropic drag coefficient field $C_{D_{\varepsilon}}^{*}$ at $\alpha=6 \mathrm{deg} / M=0.3$.

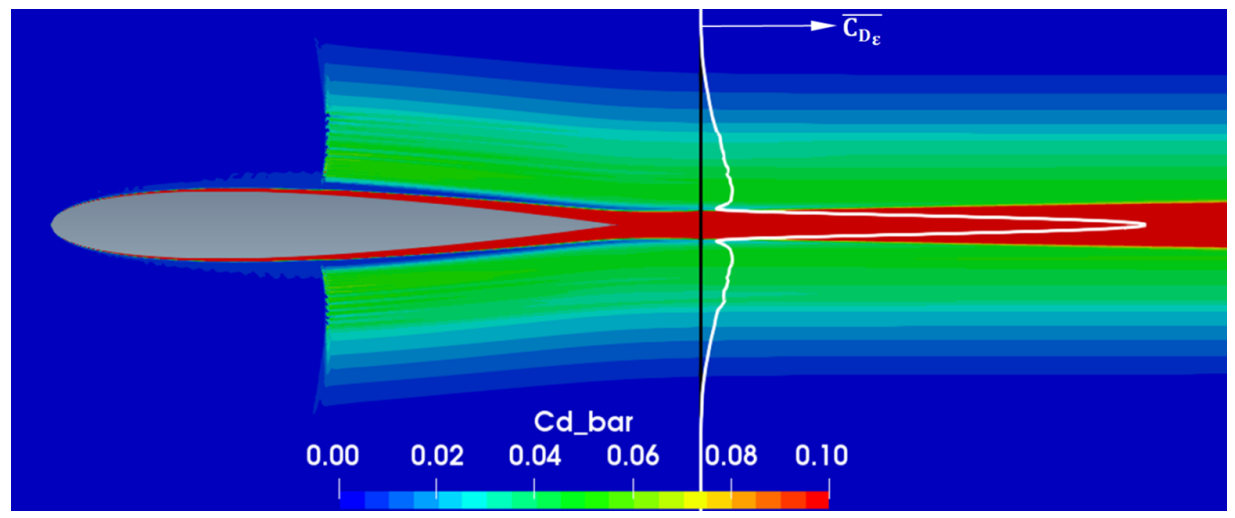

Fig. 21 Nonisentropic drag coefficient field $\overline{C_{D_{e}}}$ at $\alpha=0 \mathrm{deg} / M=0.8$.

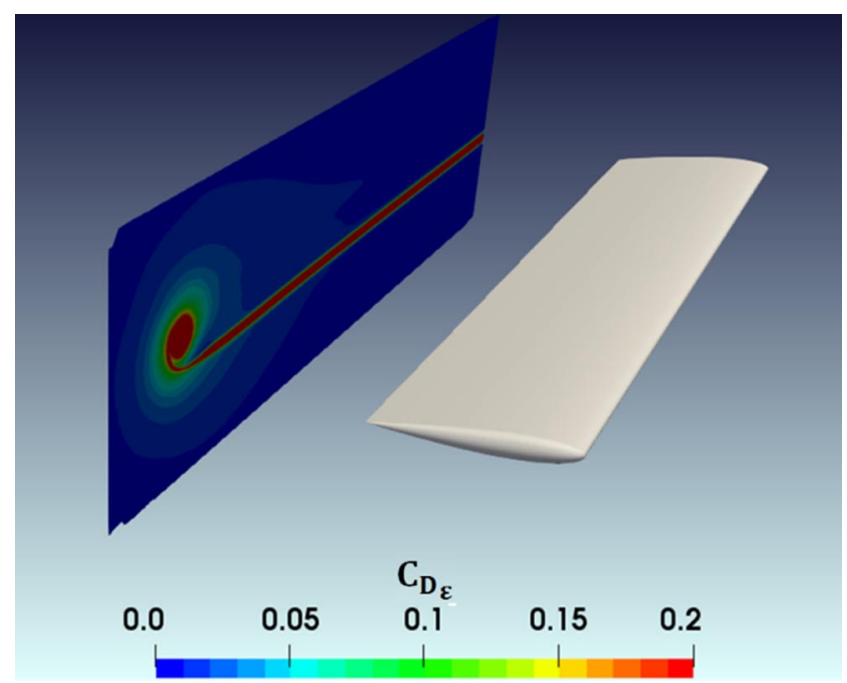

Fig. 22 Wing and survey plane at $\alpha=10 \mathrm{deg} / M=0.3$.
Then, the distributions of the components of the exergy-based drag coefficient are shown in Fig. 23. It clearly depicts that the isentropic component (Fig. 23b) takes away the viscous effects, retaining only the inviscid component. Instead, the nonisentropic component (Fig. 23c) only retains the losses and its related field is null outside the wake. Its integral will provide the profile drag but not the total drag of the body. As was explained before, the implementation of the velocity decomposition to the exergy equation will retain only the profile drag in the nonisentropic term but not the induced drag. Indeed, the induced drag is transferred to the isentropic field, as will be demonstrated later in Figs. 33 and 35. This means that the isentropic exergy-based drag coefficient field shown in Fig. $23 \mathrm{~b}$ is mostly induced drag and its field varies on the entire survey plane. However, this transverse flow is linked to the axial vorticity field shed by the body. Thus, the induced drag can be expressed as a function of wake parameters and integrated on the wake only [Eq. (18)], as has been previously proved by the Aguirre et al. [16].

\section{3-D Transonic}

In the transonic regime, the observations are similar to the subsonic case as shown in Figs. 24 and 25 . The difference here is that the 


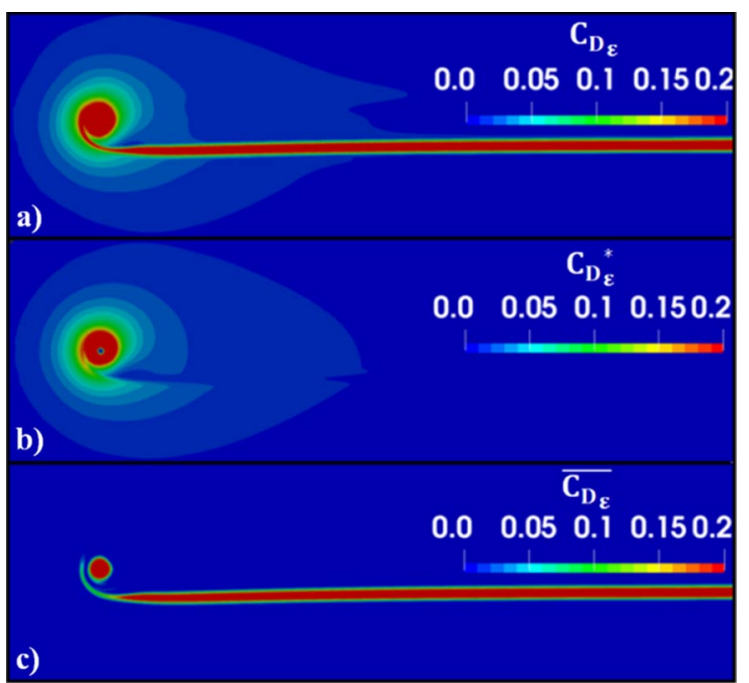

Fig. 23 Exergy-based total drag (a), induced drag (b), and profile drag (c).

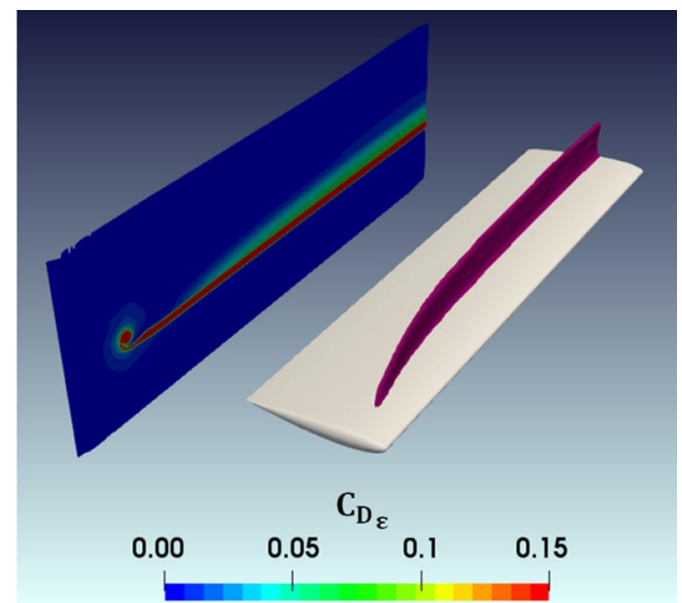

Fig. 24 Wing and survey plane at $\alpha=3 \mathrm{deg} / M=0.75$.

nonisentropic exergy-based drag coefficient (profile drag) also contains the losses associated with the shock wave. The shock wave volume is colored in violet in Fig. 24, and its wake is the green region in Fig. 25c. The wave drag can thus be extracted from this field by using the technique previously proposed by Aguirre and Duplaa [14]. Note that the angle of attack in this transonic case is smaller than the

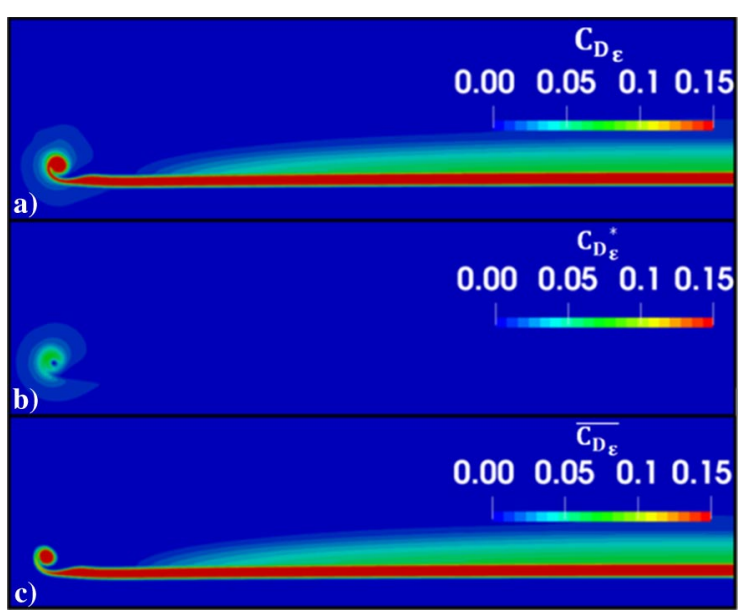

Fig. 25 Exergy-based total drag (a), induced drag (b), and profile drag (c). subsonic case presented before: that is why the intensities of the induced and profile drag distributions are weaker.

\section{Exergy Breakdown Data Analysis}

In this last section, a numerical analysis of the velocity decomposition is performed for several angles of attack. Hereafter, the survey plane used will be placed at one chord downstream of the body because it is the typical survey plane position used in windtunnel testing and because the proposed formulation is intended to be used for wind-tunnel data analysis only (although its application for CFD post-treatment is also useful for design purposes). However, it must be acknowledged that for this survey plane position, the available exergy is not at a maximum $[13,14]$, but it will be useful to present some new concepts.

\section{A. 2-D Subsonic Case}

The breakdown of the exergy-based drag coefficient [Eq. (ㅎ8)] is shown in Fig. 26. It can be seen that the nonisentropic drag $C_{\overline{D_{s}}}$ (whose integral is reduced to the wake) matches the exergy drag curve $C_{D_{\varepsilon}}$ (that requires an infinite surface integral). This highlights the usefulness of the velocity decomposition: the Arntz equations are now reduced to the wake, and a proper exergy analysis can be made with wake data from wind-tunnel testing.

On the other hand, the isentropic drag coefficient $C_{D}^{*}$ is zero or negligible in the entire range of angle of attack studied. This proves that this component does not contribute to the drag of a body (in 2-D cases) even though its field is nonzero on the entire survey plane. Indeed, the related perturbations follow a reversible transformation downstream; thus, its exergy is self-recovered and its integral is zero. It is worth mentioning that this term has been integrated on the entire survey plane. (The only parameters that can be integrated in the wake region are the nonisentropic exergies.)

To show the accuracy of the new exergy method reduced to the wake, a comparison against other methods is presented in Table 1, where far-field values (Meheut's method [7]) had required a wake integral and the Arntz method required an infinite surface integral.

Note that a very good agreement is observed: the scatter between the Arntz and the wake methods lies in the $\pm 2 \mathrm{dc}$ range. This is within the typical experimental uncertainty for drag prediction [25]. Also note the very good agreement between both wake-reduced methods: far-field and exergy. Both formulations provides the profile drag with the same order of accuracy; however, exergy analysis is even more powerful since it admits a further profile drag breakdown: the exergybased characteristic curves.

The exergy-based characteristic curves [14] are shown in Fig. 27. This decomposes the nonisentropic drag coefficient $C_{\overline{D_{\varepsilon}}}$ (profile drag) into its exergy and anergy components. The difference between the nonisentropic drag and the anergy curve $\left(C_{\overline{D_{\varepsilon}}}-C_{\dot{\mathcal{A}}}\right)$ represents

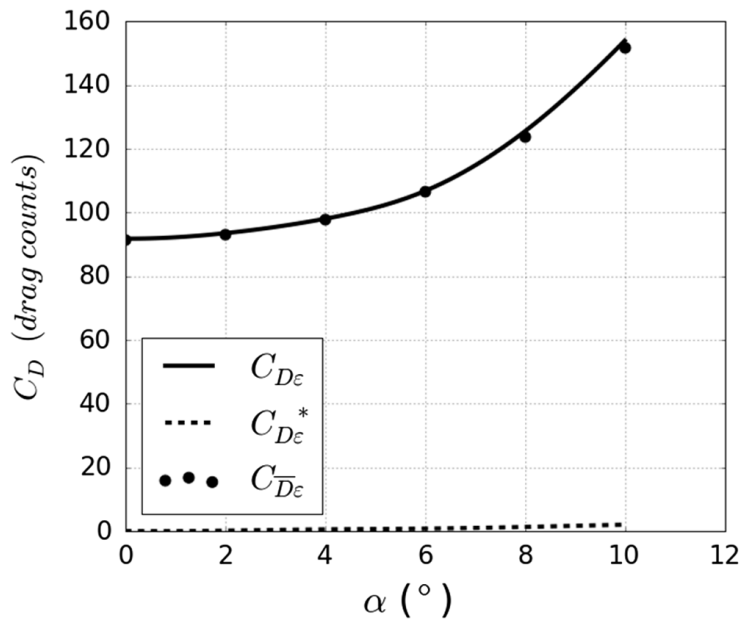

Fig. 26 Exergy drag coefficient breakdown (2D-NACA0012-M = $\left.0.3-R e=3 \times 10^{6}\right)$. 
Table 1 Profile drag coefficient values (drag counts)

\begin{tabular}{lccccc}
\hline \hline$\alpha$, deg & Near field & Far field & Exergy Arntz & Exergy wake & Wake-Arntz \\
\hline 0 & 91.58 & 91.12 & 91.80 & 91.35 & -0.45 \\
2 & 93.41 & 92.74 & 93.51 & 93.05 & -0.46 \\
4 & 99.08 & 98.14 & 98.03 & 97.60 & -0.43 \\
6 & 109.45 & 108.06 & 106.76 & 106.42 & -0.34 \\
8 & 126.2 & 124.31 & 125.48 & 123.89 & -1.59 \\
10 & 154.07 & 151.53 & 153.97 & 151.90 & -2.07 \\
\hline \hline
\end{tabular}

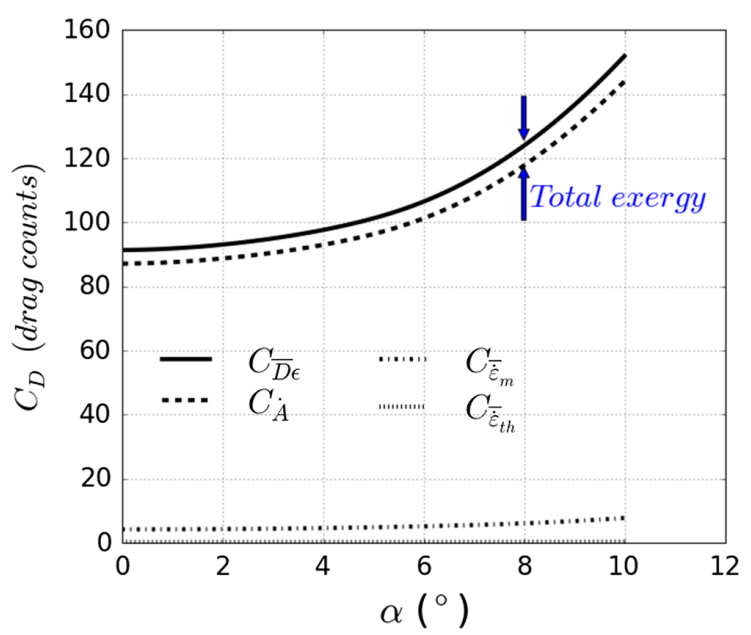

Fig. 27 Exergy characteristic curves (2D-NACA0012).

the total exergy, i.e., the maximum theoretical amount of power that can be recovered (drag reduction possibilities). Since the thermal exergy $C_{\overline{\dot{\varepsilon}}_{\mathrm{t}}}$ is negligible for external aerodynamic cases without heat transfer, this total available exergy is mainly given by the mechanical exergy $C_{\bar{\varepsilon}_{m}}$. As a reminder, the survey plane was taken one chord downstream of the body; that is why the available exergy is not that much (it is maximum at the trailing edge of the body). However, this breakdown is one of the major assets of the new method since it provides the profile drag as well as its exergy and anergy components: the physical analysis is more in-depth with the new exergy approach, leading to better design opportunities. Moreover, if closer survey plane positions are required (associated with higher exergy availability), a modified wake exergy technique must be used [17].

The previous exergy characteristic curves can be complemented by another breakdown of the isentropic and nonisentropic exergy terms (Figs. 28 and 29, respectively). The breakdown of the isentropic terms shows that the isentropic mechanical exergy $C_{\dot{\varepsilon}_{m}^{*}}$ is almost zero for 2-D cases, regardless of the angle of attack, meaning that no net energy can be recovered from this isentropic field, regardless of the fact that $C_{\dot{E}_{u}^{*}}, C_{\dot{E}_{v}^{*}}$, and $C_{\dot{E}_{p}^{*}}$ are nonzero. As a matter of fact, those terms are linked by the pressure-velocity coupling mechanisms: $C_{\dot{E}_{p}^{*}}$ compensates the existence of isentropic kinetic exergy $\left(C_{\dot{E}_{u}^{*}}+C_{\dot{E}_{v}^{*}}\right)$, leading to a net zero mechanical exergy. Also note that $C_{\dot{\varepsilon}_{\mathrm{t}}^{*}}$ is negligible. This highlights the fact that the 2-D isentropic field around a body does not contain any drag-generating mechanism (there is no net energy to be wasted downstream by entropy-generating mechanisms).

The nonisentropic components are related to viscous/shock wave phenomena, hence related to a drag generation. This drag in given by the mechanical exergy (as well as the anergy: not shown here), and

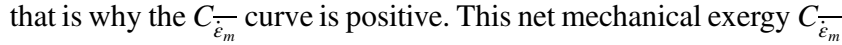
is composed by the $C_{\bar{E}_{u}}, C_{\bar{E}_{v}}$, and $C_{\bar{E}_{p}}$ fields, with $C_{\bar{E}_{v}}$ being a small quantity in 2-D cases. Note that the airfoil creates a certain amount of axial kinetic exergy $C_{\bar{E}_{u}}$ related to the axial velocity deficit in the viscous region; however, not all this exergy is potentially recoverable. In fact, part of $C_{\bar{E}_{u}}$ has been generated at the expense of $C_{\bar{E}_{p}}$. That is

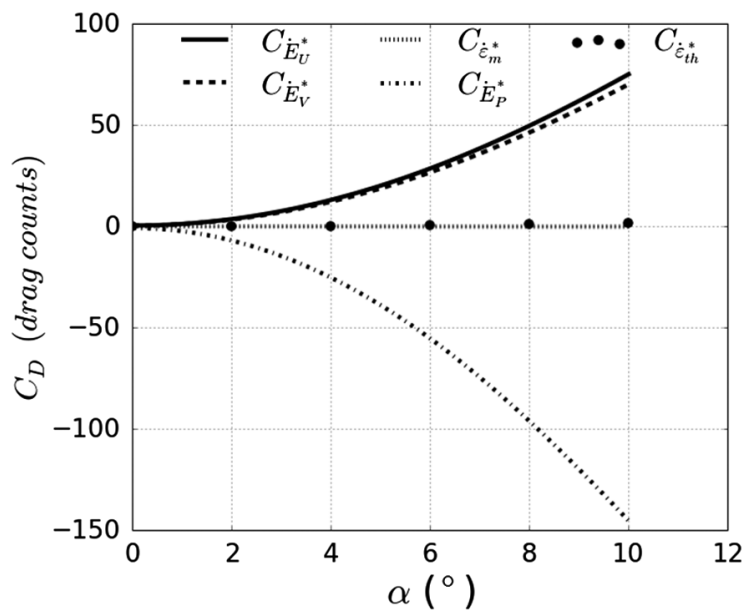

Fig. 28 Breakdown of isentropic components (2D-NACA0012).

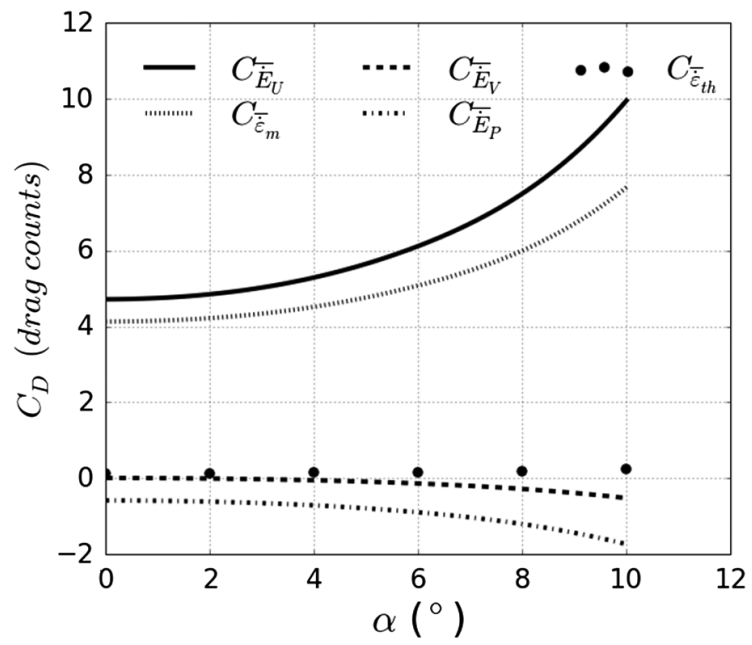

Fig. 29 Breakdown of nonisentropic components (2D-NACA0012).

why $C_{\bar{\varepsilon}_{m}}$ is the parameter that quantifies the net recoverable amount of energy. Hence, the interest of this breakdown is to pinpoint the flow topology and to get an initial guess about the type of energy recovery system to be used. In this case, a BLI system is well suited to recover $C_{\overline{\dot{\varepsilon}_{m}}}$, that is highly driven by the axial kinetic exergy. Also note that (again) the thermal exergy is negligible for this case.

All the curves in this study correspond to a survey plane position of one chord downstream of the body. The effect of moving downstream of the survey plane is shown in Figs. 30 and 31 . It can be seen that $C_{\overline{D_{s}}}$ provides the same value, regardless the survey plane position. Also, the anergy $C_{\dot{\mathcal{A}}}$ increases as the survey plane moves downstream due to the exergy destruction process (mainly by viscous dissipation along the wake). This demonstrates that the exergy is a maximum at the trailing edge of the body; hence, a BLI system must be placed closer to the airfoil trailing edge in order to recover a maximum amount of energy.

Figure 31 shows a breakdown of this net exergy $C_{\overline{\dot{\varepsilon}}}$, confirming again that the total exergy is mainly given by $C_{\bar{E}_{u}}$ (related to the velocity deficit inside the wake) for $2-\mathrm{D}$ cases. The pressure effect $C_{\bar{E}_{p}}$ is only present for closer survey plane positions due to the potential effect of the body.

The power recovery principle associated with the exergy method also leads to the introduction of a new concept: the exergy-based liftto-drag ratio $(L / D)_{\varepsilon}$, as shown in Fig. 32 . In fact, the classical lift-todrag ratio $L / D$ takes into account the profile drag for its calculation. However, since the total exergy available at the survey plane is theoretically recoverable, this profile drag could be reduced to the 


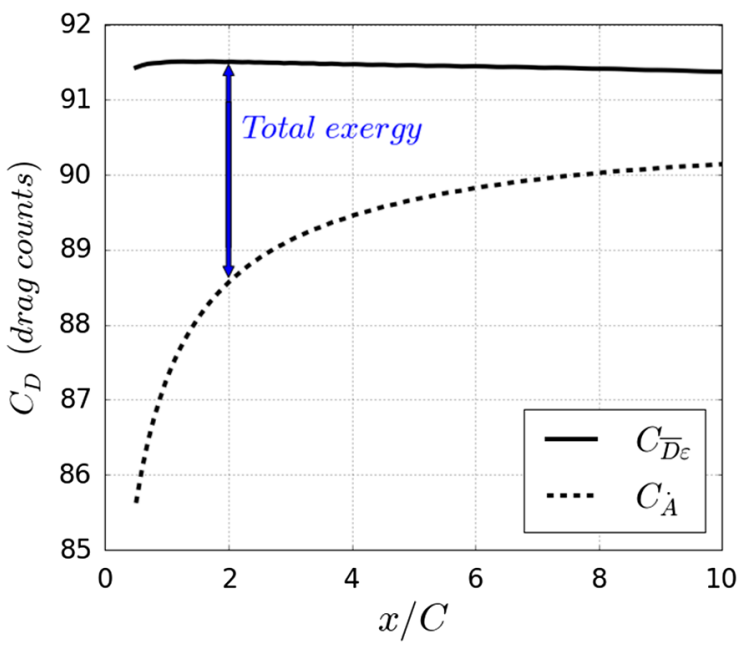

Fig. 30 Survey plane sweep downstream (2D-NACA0012).

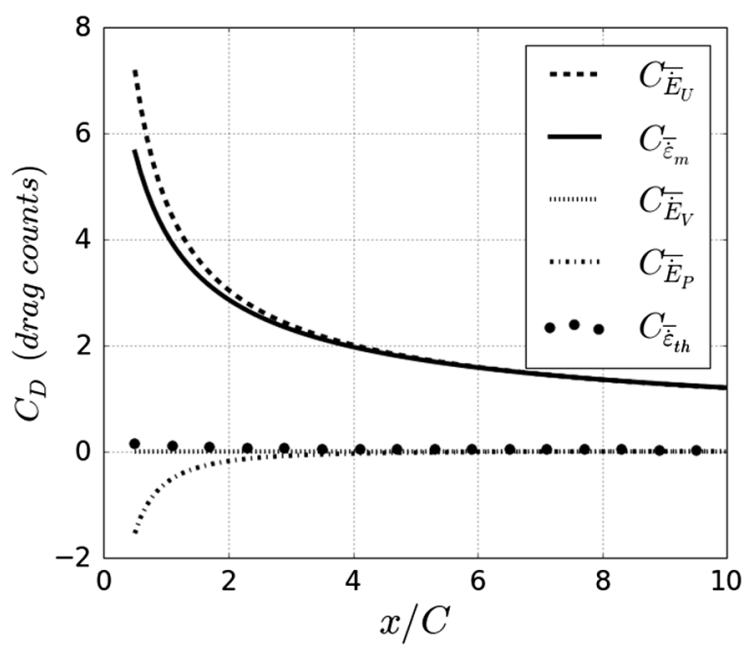

Fig. 31 Survey plane sweep downstream: breakdown (2D-NACA0012).

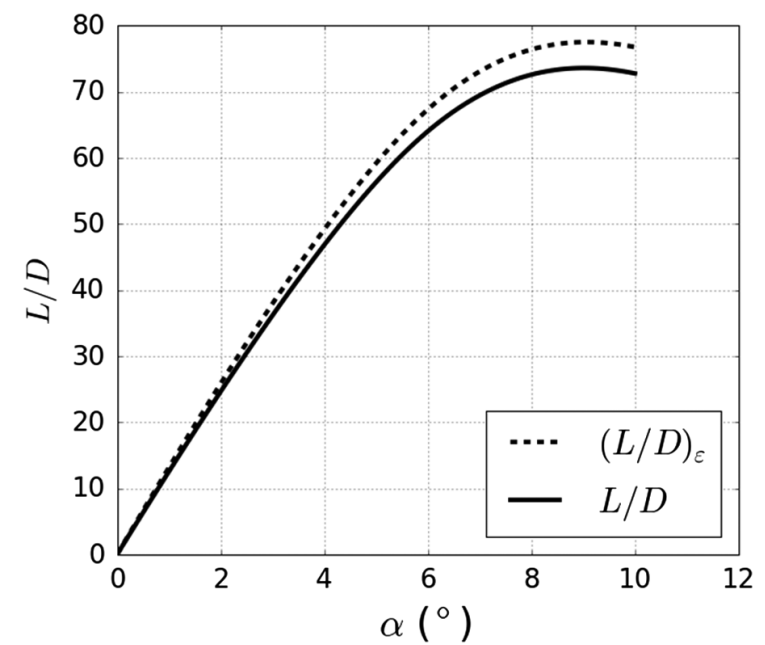

Fig. 32 Exergy-based lift-to-drag ratio (2D-NACA0012).

total anergy only; i.e., $D=\dot{\mathcal{A}}$ and the lift-to-drag ratio becomes $L / \dot{\mathcal{A}}$ or simply $(L / D)_{\varepsilon}$ in order to emphasize the fact that this is achieved by recovering the exergy. The exergy-based lift-to-drag ratio establishes the upper theoretical limit for the lift-to-drag ratio curve. This means that the actual $L / D$ of a body can be improved by recovering its exergy downstream and, if all the exergy is recovered, $L / D$

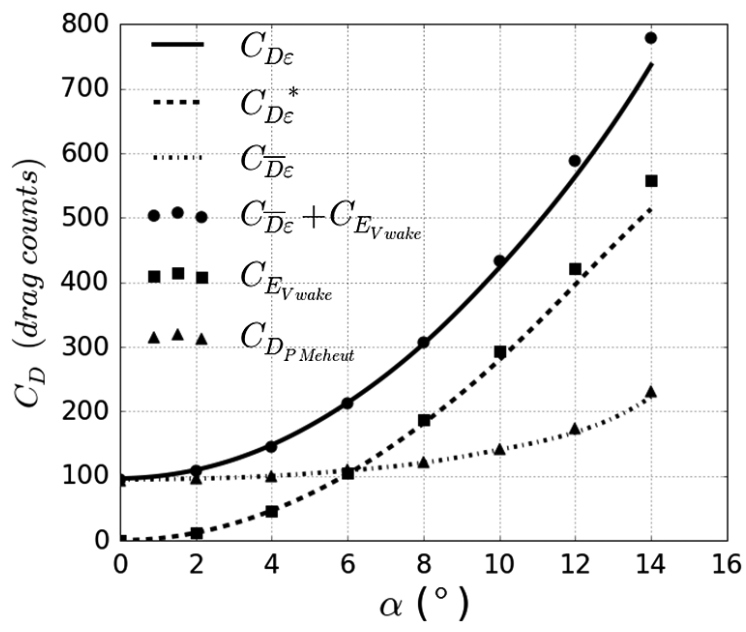

Fig. 33 Drag breakdown (3D-NACA0012 wing- $M=0.3-R e=$ $\left.3 \times 10^{6}\right)$.

reaches $(L / D)_{\varepsilon}$ : it is physically impossible (for a given geometry, flight condition, and survey plane) to overtake this limit. This parameter is very useful for design purposes because it provides a metric to know if it is worth investing a design effort to deploy energy recovery devices, depending on the remaining margin to be recovered. Here, the reader is reminded that the available exergy is at a maximum when the survey plane is closer to the body. For that plane position, the $(L / D)_{\varepsilon}$ curve will have higher values than that of Fig. 32 .

\section{B. 3-D Subsonic Case}

The results of the breakdown of the exergy formulation for a 3-D case (the NACA0012 wing) are shown in Fig. 33. The exergy-based drag coefficient $C_{D_{\varepsilon}}$ [Eq. (24)] is split into its isentropic $C_{D_{\varepsilon}}^{*}$ [Eq. (57)] and nonisentropic $\overline{C_{\overline{D_{\varepsilon}}}}$ [Eq. (58)] components. Here, the reader is reminded again that this breakdown highlights the physical origin of each component: the isentropic part is related to isentropic perturbations, whereas the nonisentropic part is related to perturbations created by entropy-generating mechanisms. Both types of perturbations are linked to drag generation for 3-D cases.

First of all, it must be noticed that the nonisentropic drag $C_{\overline{D_{\varepsilon}}}$ provides the profile drag since it is related to the viscous and shock wave losses (entropy-generating mechanisms). As a matter of fact, the $C_{\overline{D_{\varepsilon}}}$ distribution matches the Meheut profile drag curve $C_{D_{p \text { Meheut }}}$. Here, it is also worth mentioning that both the $C_{\overline{D_{\varepsilon}}}$ and the Meheut profile drag formulations have been integrated in the wake region only (both formulations are well suited for wind-tunnel testing).

Second, the isentropic curve $C_{D_{\varepsilon}}^{*}$ provides the induced drag, since this is the drag associated to the transverse rotational flowfield created by the wing, which is of isentropic origin (this rotational flowfield lies mainly in the isentropic region of the flow). Note that $C_{D_{\varepsilon}}^{*}$ matches the wake's transverse exergy curve $C_{E_{v \text { wake }}}$ [Eq. (18)]. This means that both formulations provide the induced drag but from different points of view: $C_{D \varepsilon}^{*}$ requires an infinite survey plane integral, whereas $C_{E_{v \text { wake }}}$ only requires a wake integral. Hence, in practice (wind-tunnel testing), the induced drag will be determined only by the $C_{E_{\text {vwake }}}$ method.

Finally, when the $C_{\overline{D_{s}}}$ (profile drag) is added up to $C_{E_{v \text { wake }}}$ (induced drag), the total drag can be retrieved; this total drag curve matches with the $C_{D_{\varepsilon}}$ curve. In fact, $C_{D_{\varepsilon}}$ is the total drag of a body given by the Arntz formulation, which requires an infinite survey plane integral. Instead, the sum " $C_{\overline{D_{\varepsilon}}}+C_{E_{v \text { wake }}}$ " only requires a wake integral. This can be summarized as follows:

For 3-D cases,

$$
C_{D_{\text {total }}}=C_{D_{\text {profile }}}+C_{D_{\text {induced }}}=\int_{S_{w}} C_{\overline{D_{\varepsilon}}} \mathrm{d} S+\int_{S_{w}} \frac{1}{2} \rho \psi \xi u^{*} \mathrm{~d} S
$$

This demonstrates that all the exergy parameters can be computed from wake data only, leading to a formulation suited for wind-tunnel 
Table 2 Total drag coefficient (drag counts)

\begin{tabular}{lrrrcc}
\hline \hline$\alpha$, deg & Near field & Far field & Exergy Arntz & Exergy wake & Wake-Arntz, \% \\
\hline 0 & 94.61 & 93.20 & 95.68 & 93.64 & -2.12 \\
2 & 110.58 & 107.08 & 108.94 & 106.82 & -1.95 \\
4 & 149.43 & 145.12 & 147.65 & 145.46 & -1.54 \\
6 & 213.66 & 210.97 & 212.91 & 211.96 & -0.57 \\
8 & 304.08 & 300.94 & 303.73 & 307.09 & 0.89 \\
10 & 420.28 & 417.60 & 421.44 & 432.94 & 2.43 \\
12 & 563.69 & 561.95 & 563.75 & 588.99 & 4.48 \\
14 & 736.95 & 735.47 & 736.08 & 779.55 & 5.78 \\
\hline \hline
\end{tabular}

testing. Also note that $C_{D_{\varepsilon}}^{*}$ is not an interesting parameter for windtunnel applications because it has been replaced by $C_{E_{v \text { wake }}}$ (a more practical parameter).

On the other hand, the fact that the integral of the isentropic exergybased drag $C_{D_{\varepsilon}}^{*}$ is zero in 2-D but nonzero in 3-D (and equal to the induced drag) can be misleading. However, since induced drag is zero for 2-D cases, it is reasonable to find a zero value for the integral of $C_{D_{\varepsilon}}^{*}$ in 2-D cases.

The accuracy of this new method is compared against other methods in Table 2, where the new "Exergy wake" method is given by Eq. (62). A good agreement is observed up to $\alpha=10 \mathrm{deg}$, but the error increases for larger angles of attack. To understand the source of this error, Table $\underline{3}$ shows the breakdown of the total drag into its profile and induced drag, where the Meheut method is taken as a reference. It can be observed that the profile drag given by $C_{\overline{D_{\varepsilon}}}$ provides accurate values up to $10 \mathrm{deg}$ followed by a small underprediction for larger angles of attack (but the related error is acceptable). However, it can be observed that the major source of error comes from the induced drag given by

$$
\int_{S_{w}} \frac{1}{2} \rho \psi \xi u^{*} \mathrm{~d} S
$$

This expression [16] only provides an approximation of the true induced drag given by $C_{D_{\varepsilon}}^{*}$. Further development is still required in order to improve the accuracy of this wake-reduced induced drag formulation (it is outside the scope of the present work).

The advantage of the exergy method over the far-field method is that total drag $C_{D_{\text {total }}}$ can be decomposed into its exergy and anergy components (exergy characteristic curves), as shown in Fig. 34. The difference between the total drag $\left(C_{\overline{D_{\varepsilon}}}+C_{E_{v \text { wake }}}\right)$ and the anergy $C_{\dot{\mathcal{A}}}$ gives the total exergy (predominated by the total mechanical exergy since, in an adiabatic subsonic case, the thermal exergy $C_{\bar{\varepsilon}_{\mathrm{th}}}$ is negligible). For 3-D cases, the total mechanical exergy is given by $C_{\overline{\dot{\varepsilon}_{m}}}+C_{\varepsilon \mathrm{m}}^{*}$, and represents the total amount of energy that could be theoretically recovered by a device placed downstream of the body. Note that this mechanical exergy is mostly composed by the transverse exergy $C_{E_{v \text { wake }}}$ (the axial and pressure exergies are very small in this case).

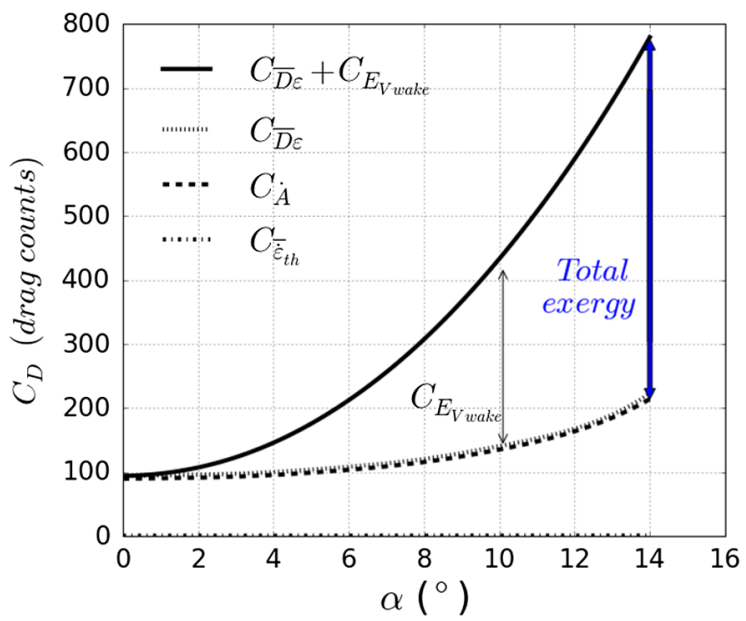

Fig. 34 Exergy characteristic curves (3-D NACA 0012 wing).

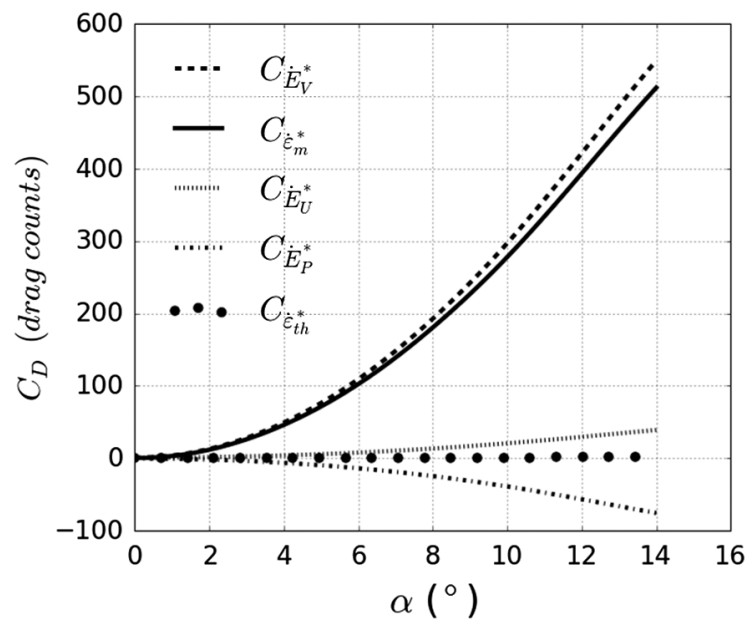

Fig. 35 Breakdown of isentropic components (3-D NACA 0012 wing).

The breakdown of the isentropic components of $C_{D^{*}}^{*}$ will shed more light on the previous analysis as shown in Fig. $\underline{35}$. In fact, $C_{\varepsilon_{m}}^{*}$ was zero for 2-D cases (see Fig. 28) but not for 3-D flows. In fact, for 2-D flows, $C_{\dot{E}_{u}^{*}}$ and $C_{\dot{E}_{v}^{*}}$ are compensated by $C_{\dot{E}_{p}^{*}}$, leading to a zero net mechanical exergy. In 3-D flows, this physical behavior is still present but coexists with an additional isentropic phenomenon: the transverse rotational flow related to the lift. Since this rotational flow field is not concentrated inside the wake region, it is not of a viscous nature, but isentropic (although it can be related to the wake information, through the axial vorticity, thanks to the $E_{v \text { wake }}$ formulation). This explains why $C_{\varepsilon_{m}}^{*}$ is not zero in 3-D flows. Moreover, since $C_{\dot{E}_{v}^{*}}$ is linked to the axial vorticity field, and this vorticity is dissipated

Table 3 Comparison of wake formulations (drag counts)

\begin{tabular}{|c|c|c|c|c|c|c|c|c|}
\hline \multirow[b]{2}{*}{$\alpha$, deg } & \multicolumn{3}{|c|}{ Far field } & \multicolumn{3}{|c|}{ Exergy wake } & \multirow[b]{2}{*}{ Profile drag new-Meheut, $\%$} & \multirow[b]{2}{*}{ Induced drag new-Meheut, $\%$} \\
\hline & Profile & Induced & Total & Profile & Induced & Total & & \\
\hline 0 & 93.20 & 0.00 & 93.20 & 93.64 & 0.00 & 93.64 & 0.47 & -0.60 \\
\hline 2 & 95.74 & 11.34 & 107.08 & 95.51 & 11.30 & 106.82 & -0.24 & -0.35 \\
\hline 4 & 99.68 & 45.44 & 145.12 & 99.67 & 45.79 & 145.46 & -0.01 & 0.77 \\
\hline 6 & 109.37 & 101.59 & 210.97 & 107.98 & 103.98 & 211.96 & -1.27 & 2.35 \\
\hline 8 & 121.55 & 179.39 & 300.94 & 119.99 & 187.10 & 307.09 & -1.28 & 4.30 \\
\hline 10 & 142.11 & 275.48 & 417.60 & 140.22 & 292.72 & 432.94 & -1.34 & 6.26 \\
\hline 12 & 173.23 & 388.71 & 561.95 & 167.32 & 421.67 & 588.99 & -3.42 & 8.48 \\
\hline 14 & 230.96 & 504.51 & 735.47 & 222.29 & 557.26 & 779.55 & -3.75 & 10.46 \\
\hline
\end{tabular}




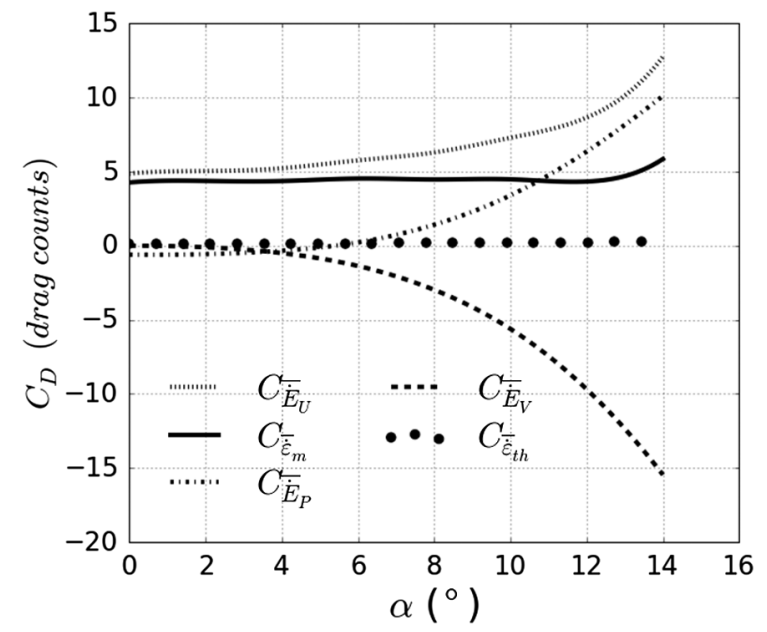

Fig. 36 Breakdown of nonisentropic components (3-D NACA 0012 wing).

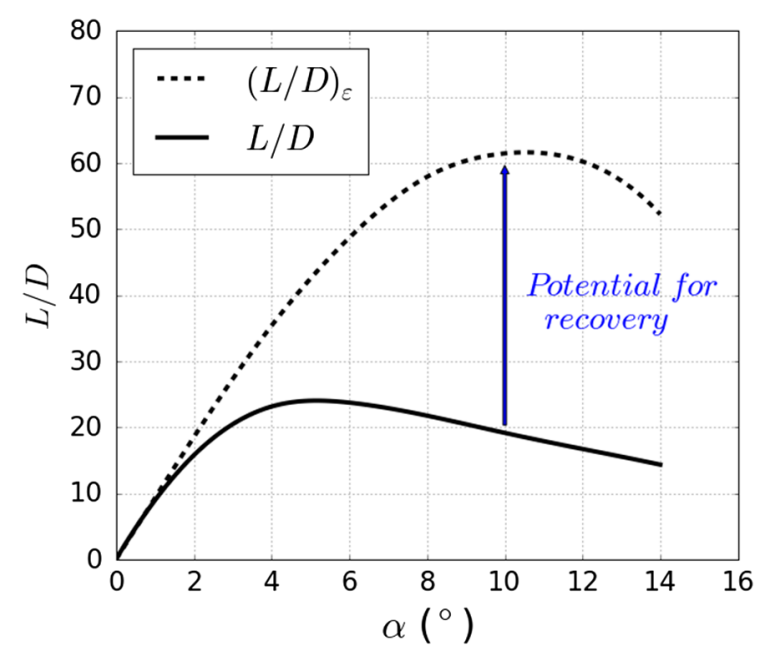

Fig. 37 Exergy-based lift-to-drag ratio.

downstream by entropy-generating mechanisms, it is clear that $C_{\dot{E}_{v}^{*}}$ will also decrease downstream (exergy destruction into anergy).

The breakdown of the nonisentropic components of $C_{\overline{D_{c}}}$ is shown in Fig. 36. The difference between this figure as compared to the 2-D case (Fig. 29) is that the existence of the wingtip vortex creates an additional pressure-velocity phenomena associated to the low pressure at the vortex core. Since the axial vorticity increases with the angle of attack, this pressure-velocity coupling is expected to increase as well.

Finally, the exergy recovery principle leads to the definition of the exergy-based lift-to-drag ratio as shown in Fig. 37. This curve shows that a significant potential for power recovery is available for 3-D cases. The difference between the $L / D$ and $(L / D)_{\varepsilon}$ curves is mainly driven by $C_{E}$ (when the survey plane is placed at one chord downstream of the body).

\section{Conclusions}

A new velocity decomposition method based on the Meheut approach was developed in order to decompose the Arntz exergy formulations. This allowed the isentropic and the nonisentropic parts of the exergy (associated with inviscid flow and the losses, respectively) to be obtained. The present work provided some major breakthroughs in the exergy formulation that are summarized as follows:

1) The velocity decomposition method, which is an improvement of the decomposition method compared to the existing methods, has been provided. The new method is more robust since the resulting isentropic contour lines shows less kink inside the viscous region.
2) For exergy breakdown, the Arntz equation has been decomposed into its isentropic and nonisentropic components, where the isentropic part gives the induced drag and the nonisentropic part provides the profile drag (in 2-D and 3-D as well).

3) For the exergy-based lift-to-drag ratio, the exergy recovery principle enables determination of the theoretical limit for the actual $L / D$ parameter. This is a useful method that establishes the margin for design improvements.

4) Regarding exergy characteristic curves from wake data, the exergy characteristic curves have been previously determined by using an infinite survey plane integral. However, the new exergy breakdown enables calculation of these parameters from wake data only. This highlights the fact that the physics driving the losses is only contained inside the wake region; thus, there is no need to analyze the entire field.

5) For the wind-tunnel formulation, since the nonisentropic equations only require a wake integral, this is well suited for wind-tunnel testing.

The main assumption of the method (isentropic velocity vector aligned with the real velocity vector) has proven to be effective for the typical aeronautical bodies with attached flow. However, this is not valid inside regions of separated flow (e.g., bluff afterbody separation bubble). In any case, the use of CFD data along with the Arntz formulation is recommended in order to verify the pertinence of this assumption for the analysis of any other geometry.

The results from this work are encouraging because they will enable further applications of the exergy method besides CFD analysis: now, the Arntz method can be extended to the experimental area. This work has also shown the advantages of the exergy method when compared against the classical methods (far field).

As a perspective, the wake-reduced formulation of the transverse exergy must be improved in order to match the isentropic exergy (induced drag).

\section{Acknowledgments}

The authors would like to thank Safran Tech and the National Higher French Institute of Aeronautics and Space for supporting this research.

\section{References}

[1] Drela, M., Flight Vehicle Aerodynamics, MIT Press, Cambridge, MA, 2014, pp. 99-112.

[2] Anderson, J., Fundamentals of Aerodynamics, McGraw-Hill Education, Boston, 2010, pp. 15-23.

[3] Betz, A., "A Method for the Direct Determination of Wing-Section Drag," NACA TR 337, 1925.

[4] Jones, B., "Measurement of Profile Drag by the Pitot-Traverse Method," Aeronautical Research Council R\&M 1688, United Kingdom, 1936.

[5] Oswatitsch, K., Gas Dynamics, Academic Press, New York, 1956.

[6] Kusunose, K., and Crowder, J. P., "Extension of Wake-Survey Analysis Method to Cover Compressible Flows," Journal of Aircraft, Vol. 39, No. 6, 2002, pp. 954-963. https://doi.org/10.2514/2.3048

[7] Meheut, M., "Evaluation des Composantes Phénoménologiques de la Trainée d'un Avion à Partir des Résultats Expérimentaux," Ph.D. Thesis, ONERA-Univ. of Sciences and Technologies of Lille, Lille, France, 2006.

[8] Riggins, D., Moorhouse, D., and Camberos, J., "Characterization of Aerospace Vehicle Performance and Mission Analysis Using Thermodynamic Availability," Journal of Aircraft, Vol. 47, No. 3, 2010, pp. 904-916. https://doi.org/10.2514/1.46420

[9] Li, H., Stewart, J., and Figliola, R., "Exergy Based Design Methodology for Airfoil Shape Optimization and Wing Analysis," 25th International Congress of the Aeronautical Sciences, ICAS, Germany, Sept. 2006, https://www.icas.org/ICAS_ARCHIVE/ICAS2006/ABSTRACTS/515 .HTM.

[10] Alabi, K., Ladeinde, F., Spakovsky, M., Moorhouse, D., and Camberos, J., "The Use of the 2nd Law as a Potential Design Tool for Aircraft Air Frame Subsystems," International Journal of Thermodynamics, Vol. 9, No. 4, 2006, pp. 193-205.

[11] Camberos, J., and Moorhouse, D., Exergy Analysis and Design Optimization for Aerospace Vehicles and Systems, Progress in Astronautics and Aeronautics, AIAA, Reston, VA, 2011.

[12] Hayes, H., Lone, M., Whidborne, J. F., Camberos, J., and Coetzee, E., "Adopting Exergy Analysis for Use in Aerospace," Progress in Aero- 
space Sciences, Vol. 93, Aug. 2017, pp. 73-94. https://doi.org/10.1016/j.paerosci.2017.07.004

[13] Arntz, A., "Civil Aircraft Aero-Thermo-Propulsive Performance Assessment by an Exergy Analysis of High-Fidelity CFD-RANS Flow Solutions," Univ. of Lille 1, Villeneuve-d'Ascq, France, 2014, https:// hal.archives-ouvertes.fr/tel-01113135.

[14] Aguirre, M., and Duplaa, S., "Exergetic Drag Characteristic Curves," AIAA Journal, Vol. 57, No. 7, 2019, pp. 2746-2757. https://doi.org/10.2514/1.J057985

[15] Aguirre, M., Duplaa, S., and Carbonneau, X., "2-D Flow Field Analysis by the Exergetic Method," AIAA Applied Aerodynamics Conference, AIAA Paper 2019-2925, June 2019. https://doi.org/10.2514/6.2019-2925

[16] Aguirre, M., Duplaa, S., and Carbonneau, X., "Vortex Exergy Prediction," 54th 3AF International Conference on Applied Aerodynamics, Association Aéronautique et Astronautique de France, Paris, France, March 2019

[17] Aguirre, M., Duplaa, S., Carbonneau, X., and Turnbull, A., "A Systematic Analysis of the Mechanical Exergy of an Airfoil by Using Potential Flow, Euler \& RANS," 24ème Congrès Français de Mécanique, Association Française de Mécanique, Brest, France, Aug. 2019.

[18] Aguirre, M., Duplaa, S., Carbonneau, X., Turnbull, A., and Arntz, A., "A Better Assessment of the Recoverable Energy Behind a Body by the Exergy Method," Aerospace Europe Conference, Association Aéronautique et Astronautique de France, Bordeaux, France, Feb. 2020.

[19] Aguirre, M., and Duplaa, S., "Epsilon: An Open Source Tool for Exergy-Based Aerodynamic Analysis," Aerospace Europe Conference,
Association Aéronautique et Astronautique de France, Bordeaux, France, Feb. 2020.

[20] Gao, A., Zou, S., Shi, Y., and Wu, J., "Energy-Based Drag Breakdown in Compressible Flow by Wake-Plane Integrals," AIAA Journal, Vol. 57, No. 8, 2019, pp. 3231-3238. https://doi.org/10.2514/1.J058114

[21] Memon, M., Wabick, K., and Altman, A., "Wing Tip Vortices from an Exergy-Based Perspective," Journal of Aircraft, Vol. 52, No. 4, 2015, pp. 1267-1276. https://doi.org/10.2514/1.C032854

[22] Cengel, Y., and Boles, M., Thermodynamics: An Engineering Approach, 8th ed., McGraw-Hill Education, New York, 2015.

[23] Bejan, A., Advanced Engineering Thermodynamics, 2nd ed., Wiley, New York, 1997.

[24] McCroskey, W., "A Critical Assessment of Wind Tunnel Results for the NACA 0012 Airfoil," NACA TM 100019, 1987.

[25] Ladson, C., "Effect of Independent Variation of Mach and Reynolds Numbers on the Low-Speed Aerodynamic Characteristics of the NACA 0012 Airfoil Section," NACA TM 4074, 1988.

[26] "Experimental Data Base for Computer Program Assessment," AGARD TR 138, Neuilly-Sur-Seine, France, 1979.

[27] Thibert, J., Grandjacques, M., and Ohman, L., "Experimental Data Base for Computer Program Assessment," AGARD TR 138, NeuillySur-Seine, France, 1979.

P. Lavoie

Associate Editor 